\title{
Nonlinear Stochastic Perturbations of Dynamical Systems and Quasi-linear Parabolic PDE's with a Small Parameter
}

\author{
M. Freidlin*, L. Koralov ${ }^{\dagger}$
}

\begin{abstract}
In this paper we describe the asymptotic behavior, in the exponential time scale, of solutions to quasi-linear parabolic equations with a small parameter at the second order term and the long time behavior of corresponding diffusion processes. In particular, we discuss the exit problem and metastability for the processes corresponding to quasi-linear initial-boundary value problems.
\end{abstract}

2000 Mathematics Subject Classification Numbers: 60F10, 35K55.

Keywords: Nonlinear Perturbations, Exit Problem, Metastability.

\section{Introduction}

Consider a dynamical system

$$
\dot{X}_{t}^{x}=b\left(X_{t}^{x}\right), \quad X_{0}^{x}=x \in \mathbb{R}^{d},
$$

together with its stochastic perturbations

$$
d X_{t}^{x, \varepsilon}=b\left(X_{t}^{x, \varepsilon}\right) d t+\varepsilon \sigma\left(X_{t}^{x, \varepsilon}\right) d W_{t}, \quad X_{0}^{x, \varepsilon}=x \in \mathbb{R}^{d} .
$$

Here $\varepsilon>0$ is a small parameter, $W_{t}$ is a Wiener process in $\mathbb{R}^{d}$, and the coefficients $\sigma$ and $b$ are assumed to be Lipschitz continuous. The diffusion matrix $a(x)=\left(a_{i j}(x)\right)=\sigma(x) \sigma^{*}(x)$ is assumed to be non-degenerate for all $x$.

Let $D$ be a bounded domain in $\mathbb{R}^{d}$ with infinitely smooth boundary $\partial D$. In this paper, with the exception of the last section, we assume that there is a point $x_{0} \in D$ such that for each $x \in D$ the trajectory of the dynamical system (11) starting at $x$ is attracted to $x_{0}$. We assume that $(b(x), n(x))<0$ for $x \in \partial D$, where $n(x)$ is the exterior normal to the

\footnotetext{
${ }^{*}$ Dept of Mathematics, University of Maryland, College Park, MD 20742, mif@math.umd.edu

${ }^{\dagger}$ Dept of Mathematics, University of Maryland, College Park, MD 20742, koralov@math.umd.edu
} 
boundary of $D$. Let $\tau^{\varepsilon}=\min \left\{t: X_{t}^{x, \varepsilon} \in \partial D\right\}$ be the first time when $X_{t}^{x, \varepsilon}$ reaches the boundary of $D$.

If $\varepsilon$ is small, then on any finite time interval the trajectories of the process $X_{t}^{x, \varepsilon}$ defined by (2) are close to the corresponding non-perturbed trajectory with probability close to one. Therefore, with high probability $X_{t}^{x, \varepsilon}$ enters a small neighborhood of the equilibrium point $x_{0}$ before leaving $D$. The process eventually exits $D$ as a result of large deviations of $X_{t}^{x, \varepsilon}$ from $X_{t}^{x}$ ([8], see also [10]). The large deviations are governed by the normalized action functional

$$
S_{0, T}(\varphi)=\frac{1}{2} \int_{0}^{T} \sum_{i, j=1}^{d} a^{i j}\left(\varphi_{t}\right)\left(\dot{\varphi}_{t}^{i}-b_{i}\left(\varphi_{t}\right)\right)\left(\dot{\varphi}_{t}^{j}-b_{j}\left(\varphi_{t}\right)\right) d t, \quad T \geq 0, \varphi \in C([0, T], \bar{D}),
$$

and the quasi-potential

$$
V\left(x_{0}, x\right)=\inf _{T, \varphi}\left\{S_{0, T}(\varphi): \varphi \in C([0, T], \bar{D}), \varphi(0)=x_{0}, \varphi(T)=x\right\}, \quad x \in \bar{D} .
$$

Here $a^{i j}$ be the elements of the inverse matrix, that is $a^{i j}=\left(a^{-1}\right)_{i j}$, and $S_{0, T}(\varphi)=+\infty$ if $\varphi$ is not absolutely continuous. It is proved in [8] that $\varepsilon^{2} \ln \tau^{\varepsilon}$ converges in probability, as $\varepsilon \downarrow 0$, to $V_{0}=\min _{x \in \partial D} V\left(x_{0}, x\right)$. Moreover, if the minimum $V_{0}$ of $V\left(x_{0}, x\right)$ on $\partial D$ is achieved at a unique point $x^{*}$ (which is true in the generic case), then $X_{\tau^{\varepsilon}}^{x, \varepsilon}$ converges to $x^{*}$ in probability as $\varepsilon \downarrow 0$.

These statements imply various results for PDE's with a small parameter at the second order derivatives. In particular, consider the following initial-boundary value problem:

$$
\begin{gathered}
\frac{\partial w^{\varepsilon}(t, x)}{\partial t}=\frac{\varepsilon^{2}}{2} \sum_{i, j=1}^{d} a_{i j}(x) \frac{\partial^{2} w^{\varepsilon}(t, x)}{\partial x_{i} \partial x_{j}}+b(x) \cdot \nabla_{x} w^{\varepsilon}(t, x), \quad x \in D, \quad t>0, \\
w^{\varepsilon}(0, x)=g(x), x \in D, \quad w^{\varepsilon}(t, x)=g(x), \quad t \geq 0, x \in \partial D,
\end{gathered}
$$

where $g$, for the sake of brevity, is assumed to be continuous on $\bar{D}$. The case when $\left.w^{\varepsilon}(t, x)\right|_{x \in \partial D}=\psi(x)$ with $\psi \neq g$ can be considered in a similar way. Assume that the minimum $V_{0}$ of $V\left(x_{0}, x\right)$ on $\partial D$ is achieved at a unique point $x^{*}$. Let $t: \mathbb{R}^{+} \rightarrow \mathbb{R}$ be a function such that $t(\varepsilon) \asymp \exp \left(\lambda / \varepsilon^{2}\right)$ as $\varepsilon \downarrow 0$ with $\lambda>0$, that is $\ln (t(\varepsilon)) \sim \lambda / \varepsilon^{2}$ as $\varepsilon \downarrow 0$. Then

$$
\begin{aligned}
& \lim _{\varepsilon \downarrow 0} w^{\varepsilon}(t(\varepsilon), x)=g\left(x_{0}\right), \quad \text { if } \lambda<V_{0}, \\
& \lim _{\varepsilon \downarrow 0} w^{\varepsilon}(t(\varepsilon), x)=g\left(x^{*}\right), \quad \text { if } \lambda>V_{0},
\end{aligned}
$$

for $x \in D$.

Note that the solution to (3) -(44) can be expressed in terms of the transition semigroup associated with the family of processes $X_{t}^{x, \varepsilon}, x \in \bar{D}$. Namely, let $T_{t}^{\varepsilon} g(x)=\mathrm{E} g\left(X_{t \wedge \tau^{\varepsilon}}^{x, \varepsilon}\right)$, $g \in C(\bar{D})$. Then the function $w^{\varepsilon}(t, x)=T_{t}^{\varepsilon} g(x)$ is the solution to (3)-(4). The semigroup $T_{t}^{\varepsilon}$ can be viewed as a small perturbation of the semigroup of shifts $T_{t} g(x)=g\left(X_{t}^{x}\right)$ associated with the dynamical system (1). 
More general perturbations of $T_{t}$ may lead to nonlinear semigroups. Namely, consider the following problem:

$$
\begin{gathered}
\frac{\partial u^{\varepsilon}(t, x)}{\partial t}=L^{\varepsilon} u^{\varepsilon}:=\frac{\varepsilon^{2}}{2} \sum_{i, j=1}^{d} a_{i j}\left(x, u^{\varepsilon}\right) \frac{\partial^{2} u^{\varepsilon}(t, x)}{\partial x_{i} \partial x_{j}}+b(x) \cdot \nabla_{x} u^{\varepsilon}(t, x), \quad x \in D, \quad t>0 \\
u^{\varepsilon}(0, x)=g(x), x \in D ; \quad u^{\varepsilon}(t, x)=g(x), \quad t \geq 0, x \in \partial D .
\end{gathered}
$$

When the coefficients are sufficiently smooth and the matrix $a$ is positive-definite, the solution $u^{\varepsilon}$ exists and is unique in the appropriate function space (see Section 2.1 below). We can now define the semigroup $T_{t}^{\varepsilon}$ on $C(\bar{D})$ via $T_{t}^{\varepsilon} g(x)=u^{\varepsilon}(t, x)$, where $u^{\varepsilon}$ is the solution of (5) - (6) with initial-boundary data $g$.

For $t>0$ and $x \in \bar{D}$, we can define $X_{s}^{t, x, \varepsilon}, s \in[0, t]$, as the process which starts at $x$ and solves

$$
\begin{gathered}
d X_{s}^{t, x, \varepsilon}=b\left(X_{s}^{t, x, \varepsilon}\right) d s+\varepsilon \sigma\left(X_{s}^{t, x, \varepsilon}, u^{\varepsilon}\left(t-s, X_{s}^{t, x, \varepsilon}\right)\right) d W_{s}, \quad s \leq \tau^{\varepsilon} \wedge t \\
\tau^{\varepsilon}=\min \left\{s: X_{s}^{t, x, \varepsilon} \in \partial D\right\}, \quad X_{s}^{t, x, \varepsilon}=X_{\tau^{\varepsilon}}^{t, x, \varepsilon}, \quad \tau^{\varepsilon} \leq s \leq t
\end{gathered}
$$

where $\sigma_{i j}, 1 \leq i, j \leq d$, are Lipschitz continuous and such that $\sigma \sigma^{*}=a$. The process $X_{s}^{t, x, \varepsilon}$ will be called the nonlinear stochastic perturbation of (11). More precisely, $X_{s}^{t, x, \varepsilon}$ corresponds to the nonlinear semigroup defined by (5). As in the linear case, we have the following relation between $u^{\varepsilon}$ and the process $X_{s}^{t, x, \varepsilon}$ :

$$
u^{\varepsilon}(t, x)=\mathrm{E} g\left(X_{t \wedge \tau^{\varepsilon}}^{t, x, \varepsilon}\right)
$$

One of the important questions in the study of parabolic linear and nonlinear equations is the one concerning the behavior of solutions (or, in probabilistic terms, behavior of the corresponding diffusion process) as $t \rightarrow \infty$. In our case, when the small parameter $\varepsilon^{2}$ is present in front of the second order term, the limit of $u^{\varepsilon}(t, x)$ as $\varepsilon \rightarrow 0, t \rightarrow \infty$, depends on the manner in which $(\varepsilon, t)$ approaches $(0, \infty)$. In the linear case this problem has been studied in [5] (see also [8], [10]).

In Section 3 we study the asymptotic behavior of solutions to (15)-(6) when $\varepsilon \downarrow 0$ and $t=t(\varepsilon) \asymp \exp \left(\lambda / \varepsilon^{2}\right)$. As a first step, we shall introduce a family of linear problems which can be obtained from (5) -(6) by replacing the second variable in the coefficients $a_{i j}$ in the right hand side of (5) by a constant $c$. The asymptotics of $u^{\varepsilon}$ can be then expressed in terms of the functions $V_{0}(c)$ and $g\left(x^{*}(c)\right)$, where $V_{0}(c)$ is the minimum of the quasi-potential of the linear problem and $x^{*}(c)$ is the point where this minimum is achieved.

In Section 4 we study the exit problem for the process $X_{s}^{t, x, \varepsilon}$. We shall see that new effects appear when nonlinear stochastic perturbations are considered. In particular, even in the generic case, the distribution of the exit location $X_{t \wedge \tau^{\varepsilon}}^{t, x, \varepsilon}$ need not be concentrated in one point.

Some related problems concern the notion of metastability for nonlinear perturbations of dynamical systems with several equilibrium points. Let us consider the dynamical 
system (11) in $\mathbb{R}^{d}$ and its perturbations (2). As before, $\sigma$ and $b$ are assumed to be Lipschitz continuous. Now we shall assume that the system has a finite number of asymptotically stable equilibrium points $x_{1}, \ldots, x_{k}$ such that for almost every $x \in \mathbb{R}^{d}$, with respect to the Lebesgue measure, the trajectory of (1) starting at $x$ is attracted to one of the points $x_{1}, \ldots x_{k}$. We shall also assume that the vector field $b$ satisfies $(b(x), x) \leq A-B|x|^{2}$ for some positive constants $A$ and $B$. The case of more general asymptotically stable attractors (for instance, limit cycles) can be considered similarly, however for the sake of brevity we restrict ourselves to the case of equilibriums.

The general theory of metastability was developed in [5] in the framework of large deviations (see also [8], [6], [10]). It was shown, in particular, that for a generic vector field $b$ satisfying the assumptions above, for almost every $x \in \mathbb{R}^{d}$ and $\lambda>0$, with probability which tends to one when $\varepsilon \downarrow 0$, the trajectory $X_{t}^{x, \varepsilon}$ of (2) spends most of the time in the time interval $\left[0, \exp \left(\lambda / \varepsilon^{2}\right)\right]$ near a point $x^{\lambda} \in\left\{x_{1}, \ldots x_{k}\right\}$. This point is called the metastable state for the trajectory starting at $x$ in the time scale $\exp \left(\lambda / \varepsilon^{2}\right)$. The metastable state can be determined by examining the values of the quasi-potential. Namely, let

$$
V_{i j}=V\left(x_{i}, x_{j}\right)=\inf _{T, \varphi}\left\{S_{0, T}(\varphi): \varphi \in C\left([0, T], \mathbb{R}^{d}\right), \varphi(0)=x_{i}, \varphi(T)=x_{j}\right\}, \quad 1 \leq i, j \leq m .
$$

These numbers determine a hierarchy of cycles along which the system switches from one metastable state to another with the growth of $\lambda([5])$.

We can also study metastability for nonlinear perturbations of dynamical systems. It turns out that now the transition between the equilibrium points does not occur "immediately in the exponential time scale". This implies that now metastable states should be replaced by metastable distributions between the equilibriums. The description of metastable distributions is based on the study of the asymptotic behavior of solutions to (5)-(6) when $\varepsilon \downarrow 0$ and $t=t(\varepsilon) \asymp \exp \left(\lambda / \varepsilon^{2}\right)$. Note that metastable distributions also arise in [2], 4], but for reasons which are different from what is discussed in this paper. Such a modification to the notion of metastability leads to a modified notion of stochastic resonance.

We briefly address the problems of metastability in Section 5 , where we also consider other generalizations and some examples. The issue of metastability in the case of an arbitrary number of equilibrium points and cycles will be addressed in a forthcoming paper.

In this paper we considered nonlinear perturbations of a system with an asymptotically stable equilibrium. In this case, the exit from a domain containing this equilibrium occurs due to large deviations, and the exit time and exit distribution essentially depend on the perturbation. A related singular perturbation problem arises in the case when the equilbrium is stable but not asymptotically stable: for instance when the unperturbed system is Hamiltonian. Nonlinear stochastic perturbations in this case lead to a nonlinear version of the averaging principle. Say, in the case of one degree of freedom, the limiting slow motion is a diffusion process corresponding to a nonlinear operator on the graph 
(compare with [8], Chapter 8) related to the Hamiltonian. We will consider these problems in one of the forthcoming papers.

\section{Preliminaries and Notations}

\subsection{Quasi-Linear Equation}

Let $D \subset \mathbb{R}^{d}$ be a bounded domain with infinitely smooth boundary $\partial D$. We shall say that $f: D \rightarrow \mathbb{R}$ belongs to $C^{2}(D)$ if $f$ and all of its partial derivatives up to the second order are bounded and continuous in $D$. We shall say that a function $f:(0, \infty) \times D \rightarrow \mathbb{R}$ belongs to $C^{1,2}((0, \infty) \times D)$ if $f$, its partial derivative in $t$, and all of its partial derivatives up to the second order in $x$ are bounded and continuous in $(0, \infty) \times D$. Note that a function $f \in C^{2}(D)$ can be extended to a continuous function on $\bar{D}$ and $f \in C^{1,2}((0, \infty) \times D)$ can be extended to a continuous function on $[0, \infty) \times \bar{D}$.

Let $a_{i j}=a_{j i} \in C^{2}(D \times \mathbb{R}), 1 \leq i, j \leq d$, and $b_{i} \in C^{2}(D), 1 \leq i \leq d$. We also assume that there is a positive constant $k$ such that $k|\xi|^{2} \leq \sum_{i, j=1}^{d} a_{i j}(x, u) \xi_{i} \xi_{j}, x \in D, u \in \mathbb{R}$, $\xi \in \mathbb{R}^{d}$. Let $g$ be an infinitely smooth function defined in a neighborhood of $\bar{D}$.

If $\varepsilon>0$ and the coefficients $a$ and $b$ and the function $g$ satisfy the assumptions listed above, then the equation (5) -(6) has a unique solution in the class of functions $C^{1,2}((0, \infty) \times D) \cap C([0, \infty) \times \bar{D})$ (see Theorem 5 , Chapter 6.2 of $\left.[9]\right)$. If $g$ were to be only continuous on $\bar{D}$, the existence and uniqueness of solutions to (5) -(6) would hold in the class of functions which are locally $C^{1,2}$-smooth inside $(0, \infty) \times D$ and continuous up to the boundary. However, to simplify notations in later sections, we impose the smoothness condition on $g$.

\subsection{Action Functional}

Let $\alpha$ be a symmetric $d \times d$ matrix whose elements $\alpha_{i j}$ are bounded and Lipschitz continuous on $\mathbb{R}^{d}$ and satisfy $k|\xi|^{2} \leq \sum_{i, j=1}^{d} \alpha_{i j}(x) \xi_{i} \xi_{j}, x \in \mathbb{R}^{d}, \xi \in \mathbb{R}^{d}$. Let $\alpha^{i j}$ be the elements of the inverse matrix, that is $\alpha^{i j}=\left(\alpha^{-1}\right)_{i j}$, and $\sigma$ be a square matrix such that $\alpha=\sigma \sigma^{*}$. We choose $\sigma$ in such a way that $\sigma_{i j}$ are also bounded and Lipschitz continuous.

Let $S_{0, T}^{\alpha}$ be the normalized action functional for the family of processes $X_{t}^{x, \varepsilon}$ satisfying

$$
d X_{t}^{x, \varepsilon}=b\left(X_{t}^{x, \varepsilon}\right) d t+\varepsilon \sigma\left(X_{t}^{x, \varepsilon}\right) d W_{t},
$$

where $b$ is a bounded Lipschitz continuous vector field on $\mathbb{R}^{d}$. Thus

$$
S_{0, T}^{\alpha}(\varphi)=\frac{1}{2} \int_{0}^{T} \sum_{i, j=1}^{d} \alpha^{i j}\left(\varphi_{t}\right)\left(\dot{\varphi}_{t}^{i}-b_{i}\left(\varphi_{t}\right)\right)\left(\dot{\varphi}_{t}^{j}-b_{j}\left(\varphi_{t}\right)\right) d t
$$

for absolutely continuous $\varphi$ defined on $[0, T], \varphi_{0}=x$, and $S_{0, T}^{\alpha}(\varphi)=\infty$ if $\varphi$ is not absolutely continuous or if $\varphi_{0} \neq x$ (see [8]). Let $V^{\alpha}(x, y)$ be the quasi-potential for the 
family $X_{t}^{x, \varepsilon}$ in $\bar{D}$, that is

$$
V^{\alpha}(x, y)=\inf _{T, \varphi}\left\{S_{0, T}^{\alpha}(\varphi): \varphi \in C([0, T], \bar{D}), \varphi(0)=x, \varphi(T)=y\right\}, \quad x, y \in \bar{D} .
$$

\section{Asymptotics of the Solution}

\subsection{Formulation of the Result}

Recall that $(b(x), n(x))<0$ for $x \in \partial D$, where $n(x)$ is the exterior normal to the boundary of $D$. We shall assume that there is an equilibrium point $x_{0} \in D$ for the vector field $b$, and that all the trajectories of the dynamical system $\dot{x}(t)=b(x(t))$ starting in $D$ are attracted to $x_{0}$. We also assume that there is $r>0$ such that $\left(b(x), x-x_{0}\right) \leq-c\left|x-x_{0}\right|^{2}$ for some positive constant $c$ and all $x$ in the $r$-neighborhood of $x_{0}$.

Let $\delta>0, D^{\delta}=\{x: x \in D$, $\operatorname{dist}(x, \partial D)>\delta\}$, and $u^{\varepsilon}$ be the solution of (15)-(16). We shall be interested in the asymptotic behavior of $u^{\varepsilon}\left(\exp \left(\lambda / \varepsilon^{2}\right), x\right)$, where $\lambda$ is fixed, $x \in D^{\delta}$, and $\varepsilon \downarrow 0$.

Let

$$
g_{\min }=\min _{x \in \bar{D}} g(x), \quad g_{\max }=\max _{x \in \bar{D}} g(x), \quad g_{1}=\min _{x \in \partial D} g(x), \quad g_{2}=\max _{x \in \partial D} g(x) .
$$

Thus $\left[g_{1}, g_{2}\right] \subseteq\left[g_{\min }, g_{\max }\right]$. Let $M:\left[g_{\min }, g_{\max }\right] \rightarrow \mathbb{R}$ be defined by

$$
M(c)=\min _{x \in \partial D} V^{a(\cdot, c)}\left(x_{0}, x\right),
$$

where $a(x, c)$ is extended to an arbitrary bounded Lipschitz continuous function satisfying $k|\xi|^{2} \leq \sum_{i, j=1}^{d} a_{i j}(x, c) \xi_{i} \xi_{j}, \xi \in \mathbb{R}^{d}, x \in \mathbb{R}^{d} \backslash D$.

We next make some assumptions about the quasi-potential. It is not difficult to see that these assumptions are satisfied by a quasi-potential corresponding to generic $a$ and $b$.

We shall assume that for all but finitely many points $c \in\left[g_{\min }, g_{\max }\right]$ the minimum in (8) is attained at a single point which will be denoted by $x^{*}(c)$. We assume that in the remaining points $c^{1}, \ldots, c^{k}$ the minimum is attained at two points of the boundary. In this case the function $x^{*}:\left[g_{\min }, g_{\max }\right] \rightarrow \partial D$ is piece-wise continuous and has left and right limits at the points of discontinuity, as follows from the formula for the quasi-potential. Let $x_{1}^{*}\left(c^{i}\right)=\lim _{c \uparrow c^{i}} x^{*}(c)$ if $c^{i} \neq g_{\min }$ and $x_{2}^{*}\left(c^{i}\right)=\lim _{c \downarrow c^{i}} x^{*}(c)$ if $c^{i} \neq g_{\max }, 1 \leq i \leq k$. If $c^{i}=g_{\min }$, we define $x_{1}^{*}\left(c^{i}\right)$ as the point distinct from $x_{2}^{*}\left(c^{i}\right)$ where the minimum of the quasi-potential is attained, and similarly we define $x_{2}^{*}\left(c^{i}\right)$ if $c^{i}=g_{\max }$ as the point distinct from $x_{1}^{*}\left(c^{i}\right)$ where the minimum of the quasi-potential is attained.

We assume that $x_{1}^{*}\left(c^{i}\right) \neq x_{2}^{*}\left(c^{i}\right), 1 \leq i \leq k$ (thus $\lim _{c \uparrow c^{i}} x^{*}(c) \neq \lim _{c \downarrow c^{i}} x^{*}(c)$ if $c^{i}$ is an interior point of $\left.\left[g_{\min }, g_{\max }\right]\right)$. Define $G_{1}\left(c^{i}\right)=g\left(x_{1}^{*}\left(c^{i}\right)\right)$ and $G_{2}\left(c^{i}\right)=g\left(x_{2}^{*}\left(c^{i}\right)\right)$. We can now define the piece-wise continuous function $G:\left[g_{\min }, g_{\max }\right] \rightarrow\left[g_{1}, g_{2}\right]$ via

$$
G(c)=g\left(x^{*}(c)\right), \quad c \in\left[g_{\min }, g_{\max }\right] \backslash\left\{c^{1}, \ldots, c^{k}\right\}, \quad G\left(c^{i}\right)=G_{1}\left(c^{i}\right), \quad 1 \leq i \leq k .
$$


Let $c_{0}=g\left(x_{0}\right)$ and define $c_{1}$ as follows:

If $G\left(c_{0}\right) \geq c_{0}$, then $c_{1}=\inf \left\{c: c \geq c_{0}, G(c) \leq c\right\}$.

If $G\left(c_{0}\right) \leq c_{0}$, then $c_{1}=\sup \left\{c: c \leq c_{0}, G(c) \geq c\right\}$.

Note that $c_{1} \in\left[g_{1}, g_{2}\right]$ since $G\left(\left[g_{\min }, g_{\max }\right]\right) \subseteq\left[g_{1}, g_{2}\right]$. We shall require that the graph of $G$ pass from the left of the diagonal to the right of the diagonal at $c_{1}$. More precisely, we shall assume that if $c_{1}>g_{\min }$, then for every $\delta_{0}>0$ there exists $\delta \in\left(0, \delta_{0}\right]$ such that

$$
G\left(c_{1}-\delta\right)>c_{1}-\delta,
$$

and if $c_{1}<g_{\max }$, then for every $\delta_{0}>0$ there exists $\delta \in\left(0, \delta_{0}\right]$ such that

$$
G\left(c_{1}+\delta\right)<c_{1}+\delta
$$

We also require that $c_{0}$ not coincide with any of the points of discontinuity $c^{i}$ for which $G_{1}\left(c^{i}\right) \leq c^{i} \leq G_{2}\left(c^{i}\right)$.

Let $\lambda \in(0, \infty)$ and define function $c(\lambda)$ as follows:

For $0<\lambda<M\left(c_{0}\right)$, let $c(\lambda)=c_{0}$.

For $\lambda \geq M\left(c_{0}\right)$ and $c_{1}=c_{0}$, let $c(\lambda)=c_{0}$.

For $\lambda \geq M\left(c_{0}\right)$ and $c_{1}>c_{0}$, let $c(\lambda)=\min \left\{c_{1}, \min \left\{c: c \in\left[c_{0}, c_{1}\right], M(c)=\lambda\right\}\right\}$.

For $\lambda \geq M\left(c_{0}\right)$ and $c_{1}<c_{0}$, let $c(\lambda)=\max \left\{c_{1}, \max \left\{c: c \in\left[c_{1}, c_{0}\right], M(c)=\lambda\right\}\right.$.

Here we use the convention that the minimum of an empty set is $+\infty$ and the maximum of an empty set is $-\infty$. (See Picture 1, where the thick line represents the graph of the function $c(\lambda)$ and $\lambda^{\prime}$ is a point of discontinuity for the function $c(\lambda)$.)

We also define $\lambda_{\max }=\sup _{c \in\left[c_{0}, c_{1}\right]} M(c)$ if $c_{1} \geq c_{0}$ and $\lambda_{\max }=\sup _{c \in\left[c_{1}, c_{0}\right]} M(c)$ if $c_{1} \leq c_{0}$.

Theorem 3.1. Let the above assumptions concerning the differential operator $L^{\varepsilon}$ and the function $G$ be satisfied. Suppose that the function $c(\lambda)$ is continuous at a point $\lambda \in(0, \infty)$. Then for every $\delta>0$ the following limit

$$
\lim _{\varepsilon \downarrow 0} u^{\varepsilon}\left(\exp \left(\lambda / \varepsilon^{2}\right), x\right)=c(\lambda)
$$

is uniform in $x \in D^{\delta}$, where $u^{\varepsilon}$ is the solution to (5)-(6).

Remark. From Theorem 3.1 and the definition of the function $c(\lambda)$ it follows that

$$
\lim _{\varepsilon \downarrow 0} u^{\varepsilon}\left(\exp \left(\lambda / \varepsilon^{2}\right), x\right)=c_{1}
$$

uniformly in $x \in D^{\delta}$ if $\lambda>\lambda_{\max }$. Moreover, from the proof of Theorem 3.1 provided below it easily follows that the limit is uniform in $(x, \lambda) \in D^{\delta} \times[\bar{\lambda}, \infty)$ for each $\bar{\lambda}>\lambda_{\max }$. Therefore, for each $\delta>0$ and $\bar{\lambda}>\lambda_{\max }$ there is $\varepsilon_{0}>0$ such that

$$
\left|u^{\varepsilon}(t, x)-c_{1}\right| \leq \delta
$$

whenever $\varepsilon \in\left(0, \varepsilon_{0}\right), x \in D^{\delta}$ and $t \geq \exp \left(\bar{\lambda} / \varepsilon^{2}\right)$. 


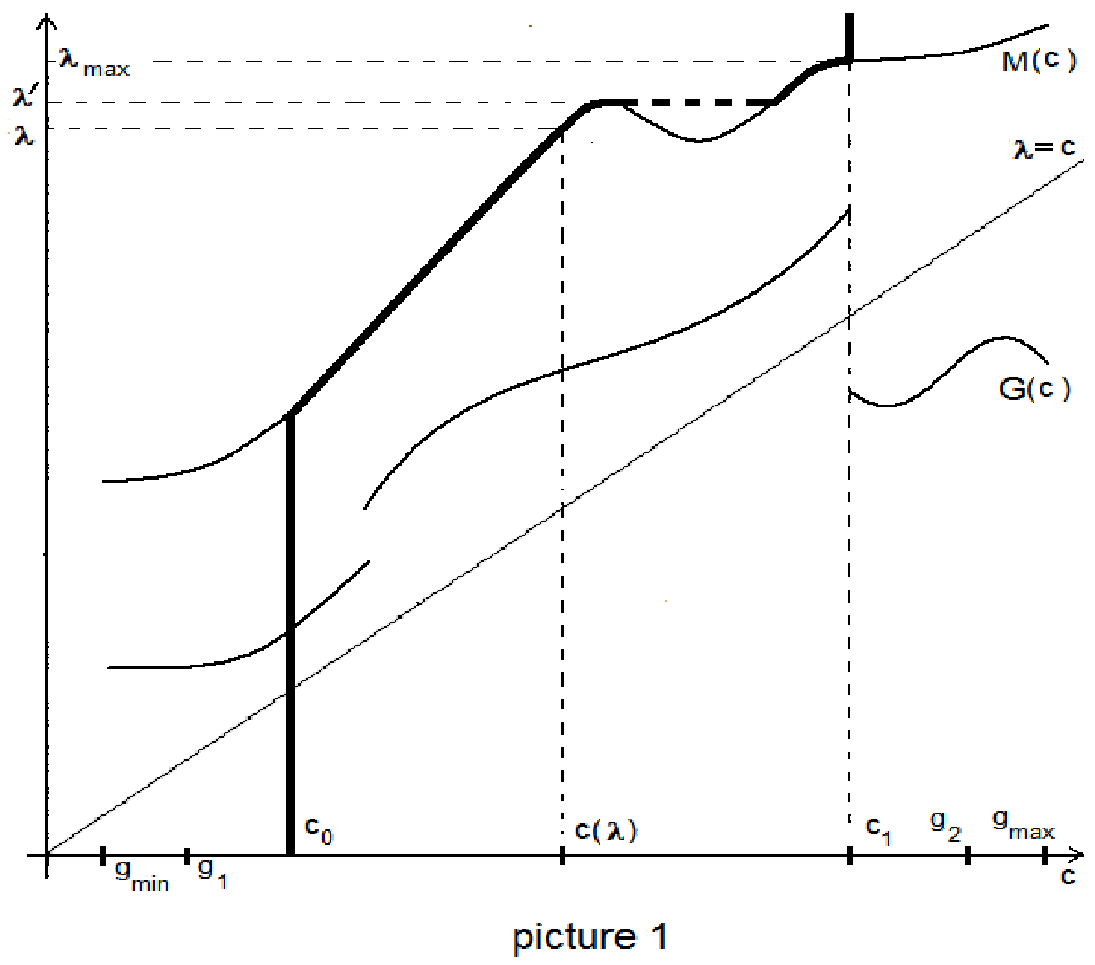

the thick line represents the graph of $c(\lambda)$

It is important to note that with the boundary values of $g$ fixed, the limit $c_{1}$ may still depend on the initial function through its value $c_{0}$ at the equilibrium point. In the generic case, when the interval $\left[g_{\min }, g_{\max }\right]$ can be represented as a finite union of intervals $I_{1} \cup \ldots \cup I_{m}$, such that on the interior of each of the intervals the function $G(c)-c$ is either strictly positive or strictly negative, the values of $c_{0}$ belonging to the interior of the same interval will correspond to the same value of $c_{1}$.

The proof of Theorem 3.1 will use some properties of diffusion processes stated in the following section. Theorem 3.1 implies various results concerning the exit problem and metastability for the process $X_{s}^{t, x, \varepsilon}$ defined above. These questions will be considered in Sections 4 and 5 .

\subsection{Properties of the Diffusion Processes}

In this section we shall consider diffusion processes which are somewhat more general than those introduced in Section 2.2. Namely, we will allow the diffusion matrix to be time dependent but close to a matrix-valued function of the space variable (that does not depend on time). The results stated in this section easily follow from the arguments of [8], Chapter 4 (see also the companion paper [7] for more details).

Let $\alpha^{\varepsilon}$ be a symmetric $d \times d$ matrix whose elements $\alpha_{i j}^{\varepsilon}$ are Lipschitz continuous on 
$\mathbb{R}^{+} \times \mathbb{R}^{d}$ and satisfy

$$
k|\xi|^{2} \leq \sum_{i, j=1}^{d} \alpha_{i j}^{\varepsilon}(t, x) \xi_{i} \xi_{j} \leq K|\xi|^{2}, \quad(t, x) \in \mathbb{R}^{+} \times \mathbb{R}^{d}, \quad \xi \in \mathbb{R}^{d},
$$

where $k$ and $K$ are positive constants. Let $\sigma^{\varepsilon}$ be a square matrix such that $\alpha^{\varepsilon}=\sigma^{\varepsilon}\left(\sigma^{\varepsilon}\right)^{*}$. We choose $\sigma^{\varepsilon}$ in such a way that $\sigma_{i j}^{\varepsilon}$ are also bounded and Lipschitz continuous.

Let $X_{t}^{x, \varepsilon}$ satisfy $X_{0}^{x, \varepsilon}=x$ and

$$
d X_{t}^{x, \varepsilon}=b\left(X_{t}^{x, \varepsilon}\right) d t+\varepsilon \sigma^{\varepsilon}\left(t, X_{t}^{x, \varepsilon}\right) d W_{t},
$$

where $b$ is a bounded Lipschitz continuous vector field on $\mathbb{R}^{d}$ satisfying the assumptions stated in Section 3.1. Clearly, the law of this process depends on $\sigma^{\varepsilon}$ only through $\alpha^{\varepsilon}=$ $\sigma^{\varepsilon}\left(\sigma^{\varepsilon}\right)^{*}$.

For $x \in D$, let $\tau^{\varepsilon}$ be the first time when the process reaches the boundary of $D$. Thus $X_{\tau^{\varepsilon}}^{x, \varepsilon}$ is the location of the first exit of the process $X_{t}^{x, \varepsilon}$ from the domain $D$. If $\alpha^{\varepsilon}$ is close to a function which does not depend on time, then the asymptotics, as $\varepsilon \downarrow 0$, of $X_{\tau^{\varepsilon}}^{x, \varepsilon}$ and $\tau^{\varepsilon}$ can be described in terms of the quasi-potential.

More precisely, let $\bar{\sigma}$ be a bounded Lipschitz continuous matrix valued function on $\mathbb{R}^{d}$ (with a Lipschitz constant $L$ ) such that

$$
k|\xi|^{2} \leq \sum_{i, j=1}^{d} \bar{\alpha}_{i j}(x) \xi_{i} \xi_{j} \leq K|\xi|^{2}, \quad x \in \mathbb{R}^{d}, \quad \xi \in \mathbb{R}^{d}
$$

where $\bar{\alpha}=\overline{\sigma \sigma}^{*}$. Let $\mathcal{A}$ be the set of points in $\partial D$ at which $\min _{x \in \partial D} V^{\bar{\alpha}}\left(x_{0}, x\right)$ is attained. This minimum will be denoted by $v$.

Lemma 3.2. Suppose $\bar{\alpha}$ is as above with a fixed Lipschitz constant L, and suppose that the positive constants $k$ and $K$ are fixed. For every $\delta>0$ there is positive $\varkappa$ and a function $\rho: \mathbb{R}^{+} \rightarrow \mathbb{R}^{+}$with $\lim _{\varepsilon \downarrow 0} \rho(\varepsilon)=0$, such that for every $\alpha^{\varepsilon}$ that is Lipschitz continuous, satisfies (9) and

$$
\sup _{(t, x) \in \mathbb{R}^{+} \times D^{\varkappa}}\left|\alpha_{i j}^{\varepsilon}(t, x)-\bar{\alpha}_{i j}(x)\right| \leq \varkappa,
$$

and every $x \in D^{\delta}$ we have:

$$
\begin{gathered}
\mathrm{P}\left(\tau^{\varepsilon} \leq \exp \left((v+\delta) / \varepsilon^{2}\right)\right) \geq 1-\rho(\varepsilon) \\
\mathrm{P}\left(\tau^{\varepsilon} \geq \exp \left((v-\delta) / \varepsilon^{2}\right)\right) \geq 1-\rho(\varepsilon) \\
\mathrm{P}\left(\operatorname{dist}\left(X_{\tau^{\varepsilon}}^{x, \varepsilon}, \mathcal{A}\right) \leq \delta\right) \geq 1-\rho(\varepsilon)
\end{gathered}
$$

The next lemma only requires the boundedness of the quadratic form $\alpha^{\varepsilon}$ from above and below.

Lemma 3.3. Suppose that positive constants $k$ and $K$ are fixed. There exists $v_{0}>0$ such that for every $0<\delta<v_{0}$ there is a function $\rho: \mathbb{R}^{+} \rightarrow \mathbb{R}^{+}$with $\lim _{\varepsilon \downarrow 0} \rho(\varepsilon)=0$, such that for every $\alpha^{\varepsilon}$ that is Lipschitz continuous and satisfies (9) and every $x \in D^{\delta}$ we have: 


$$
\begin{gathered}
\mathrm{P}\left(\tau^{\varepsilon} \geq \exp \left(v_{0} / \varepsilon^{2}\right)\right) \geq 1-\rho(\varepsilon), \\
\inf _{t \in\left[\exp \left(\delta / \varepsilon^{2}\right) \exp \left(v_{0} / \varepsilon^{2}\right)\right]} \mathrm{P}\left(\left|X_{t}^{x, \varepsilon}-x_{0}\right| \leq \delta\right) \geq 1-\rho(\varepsilon), \\
\inf _{t \in\left[0, \exp \left(v_{0} / \varepsilon^{2}\right)\right]} \mathrm{P}\left(\left|X_{t}^{x_{0}, \varepsilon}-x_{0}\right| \leq \delta\right) \geq 1-\rho(\varepsilon) .
\end{gathered}
$$

An easy corollary of this lemma is that at an exponential time the process either can be found in a small neighborhood of $x_{0}$ or has earlier crossed the boundary of the domain.

Corollary 3.4. Suppose that positive constants $k$ and $K$ are fixed. For every $\delta>0$ there is a function $\rho: \mathbb{R}^{+} \rightarrow \mathbb{R}^{+}$with $\lim _{\varepsilon \downarrow 0} \rho(\varepsilon)=0$, such that for every $\alpha^{\varepsilon}$ that is Lipschitz continuous and satisfies (9), every $x \in D$ and $t \geq \exp \left(\delta / \varepsilon^{2}\right)$ we have:

$$
\mathrm{P}\left(\left|X_{t}^{x, \varepsilon}-x_{0}\right| \leq \delta \text { or } \tau^{\varepsilon} \leq t\right) \geq 1-\rho(\varepsilon) .
$$

Proof. Let $\delta_{1}>0$ be sufficiently small so that there is a domain $\widetilde{D}$ with smooth boundary such that $\widetilde{D}^{\delta_{1}}=D$. If the process does not reach $\partial D$ by the time $t-\exp \left(\delta / \varepsilon^{2}\right)$, then we can apply Part (B) of Lemma 3.3 to the domain $\widetilde{D}$ and the process starting at $X_{t-\exp \left(\delta / \varepsilon^{2}\right)}^{x,}$, and the result follows from the Markov property.

\subsection{Preliminary Lemmas}

The next step in the proof of Theorem 3.1 is to establish that $u^{\varepsilon}\left(\exp \left(t / \varepsilon^{2}\right), x\right)$ is nearly constant on $D^{\delta}$ if $t>0$ is fixed and $\varepsilon$ is sufficiently small. This is accomplished in Lemma 3.8 below.

Lemma 3.5. For every positive $t_{0}$ and $\delta$ there are positive $R$ and $\varepsilon_{0}$ such that

$$
\left|u^{\varepsilon}(t, x)-u^{\varepsilon}\left(t, x_{0}\right)\right| \leq \delta
$$

whenever $\left|x-x_{0}\right| \leq R \varepsilon, \varepsilon \leq \varepsilon_{0}$ and $t \geq t_{0}$.

Proof. Let $v^{\varepsilon}(t, y)=u^{\varepsilon}\left(t, x_{0}+\varepsilon y\right), t \in(0, \infty),|y| \leq 2$. Let $Q=(0, \infty) \times B_{2}(0)$ and $Q_{0}=\left(t_{0}, \infty\right) \times B_{1}(0)$, where $B_{r}(0)$ is the ball of radius $r$ centered at the origin. Then $v^{\varepsilon}$ satisfies the following partial differential equation:

$$
\frac{\partial v^{\varepsilon}(t, y)}{\partial t}=\frac{1}{2} \sum_{i, j=1}^{d} \widetilde{a}_{i j}(t, y) \frac{\partial^{2} v^{\varepsilon}(t, y)}{\partial y_{i} \partial y_{j}}+\frac{b\left(x_{0}+\varepsilon y\right)}{\varepsilon} \cdot \nabla_{y} v^{\varepsilon}(t, y), \quad(t, y) \in Q .
$$

Here $\widetilde{a}_{i j}(t, y)=a_{i j}\left(x_{0}+\varepsilon y, u^{\varepsilon}\left(t, x_{0}+\varepsilon y\right)\right)$ are uniformly bounded in $\varepsilon$ and satisfy $k|\xi|^{2} \leq$ $\sum_{i, j=1}^{d} \widetilde{a}_{i j}(t, y) \xi_{i} \xi_{j}, t \in(0, \infty), y \in B_{2}(0), \xi \in \mathbb{R}^{d}$. Moreover, $\sup _{y \in B_{2}(0)}\left|b\left(x_{0}+\varepsilon y\right) / \varepsilon\right|$ is bounded uniformly in $\varepsilon$ and $\left|\nabla_{y} \widetilde{a}_{i j}(t, y)\right|$ can be estimated from above by a constant times $1+\left|\nabla_{y} v^{\varepsilon}(t, y)\right|$ for $(t, y) \in Q$, uniformly in $\varepsilon$. Since the distance between $Q_{0}$ and the boundary of $Q$ is positive and $\left|v^{\varepsilon}\right|$ is uniformly bounded in $Q$ by $\max \left(\left|g_{1}\right|,\left|g_{2}\right|\right)$, we can apply the a priori estimate (see Theorem 4, Chapter 5.2 of [9] or Theorem 6, Chapter 6.2 of [9]) to bound $\sup _{(t, x) \in Q_{0}}\left|\nabla_{y} v^{\varepsilon}\right|$ by a constant $C$ independent of $\varepsilon$. This implies that 
$\left|u^{\varepsilon}\left(t, x_{0}+\varepsilon y\right)\right|$ is bounded by $C R$ when $|y| \leq R$ if $R$ is a positive constant. It remains to take $R$ such that $C R \leq \delta$.

We'll need the following simple lemma about diffusion processes with the drift directed towards the origin.

Lemma 3.6. Let $\widetilde{b}$ be a $C^{2}$ smooth vector field on $\mathbb{R}^{d}$ such that $(\widetilde{b}(x), x) \leq-k_{1}(x, x)$ for some positive $k_{1}$ and all $x \in \mathbb{R}^{d}$. Let $\sigma^{\varepsilon}(t, x)$ be a Lipschitz continuous function such that $\alpha^{\varepsilon}=\sigma^{\varepsilon}\left(\sigma^{\varepsilon}\right)^{*}$ satisfies (9). Let $Y_{t}^{x, \varepsilon}$ be the process starting at $x$ that satisfies

$$
d Y_{t}^{x, \varepsilon}=\widetilde{b}\left(Y_{t}^{x, \varepsilon}\right) d t+\varepsilon \sigma^{\varepsilon}\left(t, Y_{t}^{x, \varepsilon}\right) d W_{t} .
$$

Then for every $r, R>0$ there are positive $\gamma, s_{0}$, and $\varepsilon_{0}$, which depend on $\widetilde{b}$ and $\sigma^{\varepsilon}$ only through $k_{1}, k$, and $K$ such that

$$
\mathrm{P}\left(Y_{s_{0}|\ln \varepsilon|}^{x, \varepsilon} \in B_{R \varepsilon}(0)\right) \geq \gamma
$$

holds for $x \in B_{r}(0)$ and $0<\varepsilon \leq \varepsilon_{0}$.

Proof. First, let us show that the probability that the process enters a larger ball in time $s_{0}|\ln \varepsilon|-1$ is bounded from below. Let $h: \mathbb{R} \rightarrow[0,1]$ be a smooth even function with negative derivative on $(1 / 2,1)$, such that $h(x)=1$ for $0 \leq x \leq 1 / 2$ and $h(x)=0$ for $x \geq 1$. Let

$$
f(t, x)=h\left(|x| \exp \left(-2 k_{1} t\right) / R_{1} \varepsilon\right),
$$

where $R_{1}>0$ will be specified below. If $R_{1}$ and $s_{0}$ are sufficiently large and $\varepsilon_{0}$ is sufficiently small, then $f\left(s_{0}|\ln \varepsilon|-1, x\right)=1$ for $x \in B_{r}(0), 0<\varepsilon \leq \varepsilon_{0}$. By the Ito formula,

$$
\begin{gathered}
\mathrm{P}\left(Y_{s_{0}|\ln \varepsilon|-1}^{x, \varepsilon} \in B_{R_{1} \varepsilon}(0)\right) \geq \operatorname{E} f\left(0, Y_{s_{0}|\ln \varepsilon|-1}^{x, \varepsilon}\right)= \\
\operatorname{Ef}\left(s_{0}|\ln \varepsilon|-1, x\right)+\mathrm{E} \int_{0}^{s_{0}|\ln \varepsilon|-1}\left(\mathcal{L}^{\varepsilon} f-\frac{\partial f}{\partial t}\right)\left(s_{0}|\ln \varepsilon|-1-s, Y_{s}^{x, \varepsilon}\right) d s,
\end{gathered}
$$

where $\mathcal{L}^{\varepsilon}$ is the generator of the process $Y_{t}^{x, \varepsilon}$. In order to estimate the integral in the right hand side, we note that

$$
\left(\mathcal{L}^{\varepsilon} f-\frac{\partial f}{\partial t}\right)(t, x) \geq-C \max _{x \in[0,1]}\left(h^{\prime \prime}(x)\right) \exp \left(-2 k_{1} t\right) / R_{1}^{2},
$$

where the constant $C$ depends on $k_{1}$ and $K$. By taking $R_{1}$ sufficiently large, we can bound the expectation of the integral from below by $-1 / 2$. We have thus demonstrated that

$$
\mathrm{P}\left(Y_{s_{0}|\ln \varepsilon|-1}^{x, \varepsilon} \in B_{R_{1} \varepsilon}(0)\right) \geq 1 / 2
$$

holds for $x \in B_{r}(0)$ and $0<\varepsilon \leq \varepsilon_{0}$. This, together with the Markov property, will imply (14) if we show that there is $\gamma>0$ such that

$$
\mathrm{P}\left(Y_{1}^{x, \varepsilon} \in B_{R \varepsilon}(0)\right) \geq 2 \gamma
$$


holds for $x \in B_{R_{1} \varepsilon}(0)$ and $0<\varepsilon \leq \varepsilon_{0}$. The latter inequality is a consequence of the Aronson estimate ([1]). Indeed, we can make the same change the variables in the generator of the process as in the proof of Lemma 3.5, thus obtaining a uniformly elliptic operator, and therefore the Aronson estimate is applicable.

Lemma 3.7. Let the process $X_{t}^{x, \varepsilon}$ satisfy (10) with the coefficients satisfying the assumptions stated in Section 3.2. Suppose that positive constants $k, K$, and $R$ are fixed. There exist $\gamma>0$ and $v_{0}>0$ such that for every $0<\delta<v_{0}$ there is $\varepsilon_{0}>0$, such that for every $\alpha^{\varepsilon}$ that is Lipschitz continuous and satisfies (9) and every $x \in D^{\delta}$ we have:

$$
\inf _{t \in\left[\exp \left(\delta / \varepsilon^{2}\right), \exp \left(v_{0} / \varepsilon^{2}\right)\right]} \mathrm{P}\left(\left|X_{t}^{x, \varepsilon}-x_{0}\right| \leq R \varepsilon\right) \geq \gamma
$$

when $\varepsilon \leq \varepsilon_{0}$.

Proof. This lemma easily follows from Lemmas 3.3, 3.6, and the Markov property of the process.

Finally, we show that the function $u^{\varepsilon}$ is nearly constant on the domain for each fixed, sufficiently large, value of time.

Lemma 3.8. For every positive $\lambda_{0}$ and $\delta$ there is positive $\varepsilon_{0}$ such that

$$
\left|u^{\varepsilon}\left(\exp \left(\lambda / \varepsilon^{2}\right), x\right)-u^{\varepsilon}\left(\exp \left(\lambda / \varepsilon^{2}\right), x_{0}\right)\right| \leq \delta
$$

whenever $x \in D^{\delta}, \varepsilon \leq \varepsilon_{0}$ and $\lambda \geq \lambda_{0}$.

Proof. Take $t_{0}=1$ and find $\varepsilon_{0}$ and $R$ such that (13) holds with $\delta / 4$ instead of $\delta$ in the right hand side, provided that $\left|x-x_{0}\right| \leq R \varepsilon, \varepsilon \leq \varepsilon_{0}$ and $t \geq t_{0}$. By Lemma 3.7 we can take $\gamma>0$ and $v_{0}<\lambda_{0}$ such that for each $\delta^{\prime}>0$ we have

$$
\mathrm{P}\left(\left|X_{\exp \left(v_{0} / \varepsilon^{2}\right)}^{x, \varepsilon}-x_{0}\right| \leq R \varepsilon\right) \geq \gamma
$$

for all $x \in D^{\delta^{\prime}}$ and all sufficiently small $\varepsilon$. Observe that

$$
\sup _{t \geq 0, x, y \in D}\left|u^{\varepsilon}(t, x)-u^{\varepsilon}(t, y)\right| \leq \sup _{x, y \in D}|g(x)-g(y)| .
$$

Choose an integer

$$
N \geq 1+6 \sup _{x, y \in D}|g(x)-g(y)| /(\delta \gamma)
$$

and consider the sequence of domains $D^{\delta} \subset D^{\delta / 2} \subset \ldots \subset D^{\delta / N}$. Let

$$
q_{m}=\sup _{x, y \in D^{\delta / m}}\left|u^{\varepsilon}\left(e^{\frac{\lambda}{\varepsilon^{2}}}-(m-1) e^{\frac{v_{0}}{\varepsilon^{2}}}, x\right)-u^{\varepsilon}\left(e^{\frac{\lambda}{\varepsilon^{2}}}-(m-1) e^{\frac{v_{0}}{\varepsilon^{2}}}, y\right)\right| .
$$

We claim that

$$
q_{m} \leq\left(1-\frac{\gamma}{2}\right) q_{m+1}+\frac{\gamma \delta}{3}, \quad m=1, \ldots, N
$$


for all sufficiently small $\varepsilon$. Since $q_{N} \leq \sup _{x, y \in D}|g(x)-g(y)|$, the inequalities (17) and (18) imply that $q_{1} \leq \delta$, which gives (16). It remains to prove (18).

We suppress the dependence on $m$ and $\varepsilon$ in the notation for the process $Y_{s}^{x}=Y_{s}^{m, x, \varepsilon}$, $s \in\left[0, \exp \left(v_{0} / \varepsilon^{2}\right)\right]$, that starts at $x \in D^{\delta / m}$ and satisfies

$$
\begin{gathered}
d Y_{s}^{x}=b\left(Y_{s}^{x}\right) d s+\varepsilon \sigma\left(Y_{s}^{x}, u^{\varepsilon}\left(e^{\frac{\lambda}{\varepsilon^{2}}}-(m-1) e^{\frac{v_{0}}{\varepsilon^{2}}}-s, Y_{s}^{x}\right)\right) d W_{s}, \quad s \leq \tau^{x} \wedge \exp \left(v_{0} / \varepsilon^{2}\right) \\
\tau^{x}=\min \left\{s: Y_{s}^{x} \in \partial D^{\delta /(m+1)}\right\}, \quad Y_{s}^{x}=Y_{\tau^{x}}^{x}, \quad \tau^{x} \leq s \leq \exp \left(v_{0} / \varepsilon^{2}\right)
\end{gathered}
$$

where $\sigma_{i j} \in C^{2}(D \times \mathbb{R}), 1 \leq i, j \leq d$, are Lipschitz continuous and such that $\sigma \sigma^{*}=a$. (If the minimum in the definition of $\tau^{x}$ is taken over an empty set, then it is considered to be equal to $+\infty$.)

Let $\Omega$ denote the probability space on which the diffusion process is defined, and consider its partition into the following disjoint events:

$$
\begin{gathered}
G_{1}^{x}=\left\{\left|Y_{\exp \left(v_{0} / \varepsilon^{2}\right)}^{x}-x_{0}\right| \leq R \varepsilon, \quad \tau^{x} \geq \exp \left(v_{0} / \varepsilon^{2}\right)\right\}, \\
G_{2}^{x}=\left\{\left|Y_{\exp \left(v_{0} / \varepsilon^{2}\right)}^{x}-x_{0}\right|>R \varepsilon, \quad \tau^{x} \geq \exp \left(v_{0} / \varepsilon^{2}\right)\right\}, \\
G_{3}^{x}=\left\{\tau^{x}<\exp \left(v_{0} / \varepsilon^{2}\right)\right\} .
\end{gathered}
$$

From our construction and Lemma 3.3 it follows that $\mathrm{P}\left(G_{1}^{x}\right) \geq \gamma / 2$ for all sufficiently small $\varepsilon$. Let us take an arbitrary event $F_{1}^{x} \subseteq G_{1}^{x}$ such that $\mathrm{P}\left(F_{1}^{x}\right)=\gamma / 2$. From Lemma 3.3 it follows that $\mathrm{P}\left(G_{3}^{x}\right) \leq \gamma \delta /\left(1+24 \sup _{x, y \in D}|g(x)-g(y)|\right)$ for all sufficiently small $\varepsilon$. We can assume that $\gamma$ is sufficiently small so that $\gamma \delta /\left(1+24 \sup _{x, y \in D}|g(x)-g(y)|\right)+\gamma / 2<1$. Let us take an arbitrary event $F_{3}^{x}$ such that $\mathrm{P}\left(F_{3}^{x}\right)=\gamma \delta /\left(1+24 \sup _{x, y \in D}|g(x)-g(y)|\right)$ and $G_{3}^{x} \subseteq F_{3}^{x} \subseteq \Omega \backslash F_{1}^{x}$. Let $F_{2}^{x}=\Omega \backslash\left(F_{1}^{x} \cup F_{3}^{x}\right)$.

By the Feynman-Kac formula,

$$
\begin{gathered}
u^{\varepsilon}\left(e^{\frac{\lambda}{\varepsilon^{2}}}-(m-1) e^{\frac{v_{0}}{\varepsilon^{2}}}, x\right)=\mathrm{E} u^{\varepsilon}\left(e^{\frac{\lambda}{\varepsilon^{2}}}-(m-1) e^{\frac{v_{0}}{\varepsilon^{2}}}-\tau^{x}, Y_{\tau^{x}}^{x}\right)= \\
\mathrm{E}\left[u^{\varepsilon}\left(e^{\frac{\lambda}{\varepsilon^{2}}}-(m-1) e^{\frac{v_{0}}{\varepsilon^{2}}}-\tau^{x}, Y_{\tau^{x}}^{x}\right) \chi_{F_{1}^{x}}\right]+\mathrm{E}\left[u^{\varepsilon}\left(e^{\frac{\lambda}{\varepsilon^{2}}}-(m-1) e^{\frac{v_{0}}{\varepsilon^{2}}}-\tau^{x}, Y_{\tau^{x}}^{x}\right) \chi_{F_{2}^{x}}\right]+ \\
\mathrm{E}\left[u^{\varepsilon}\left(e^{\frac{\lambda}{\varepsilon^{2}}}-(m-1) e^{\frac{v_{0}}{\varepsilon^{2}}}-\tau^{x}, Y_{\tau^{x}}^{x}\right) \chi_{F_{3}^{x}}\right]=I_{1}^{x}+I_{2}^{x}+I_{3}^{x} .
\end{gathered}
$$

Therefore,

$$
q_{m} \leq \sup _{x, y \in D_{m}}\left(\left|I_{1}^{x}-I_{1}^{y}\right|+\left|I_{2}^{x}-I_{2}^{y}\right|+\left|I_{3}^{x}-I_{3}^{y}\right|\right)
$$

The term $\left|I_{1}^{x}-I_{1}^{y}\right|$ is estimated from above by $\gamma \delta / 4$ since the value of the function $u^{\varepsilon}$ inside the $R \varepsilon$ neighborhood of $x_{0}$ doesn't vary by more than $\delta / 2$. The term $\left|I_{2}^{x}-I_{2}^{y}\right|$ can be estimated from above by $(1-\gamma / 2) q_{m+1}$ since $\mathrm{P}\left(F_{2}^{x}\right)=\mathrm{P}\left(F_{2}^{y}\right) \leq 1-\gamma / 2$. Similarly, the term $\left|I_{3}^{x}-I_{3}^{y}\right|$ is estimated from above by $\gamma \delta / 12$. Combining the terms, yields (18), which completes the proof of the lemma. 


\subsection{Proof of the Theorem on the Asymptotics of the Solution}

In this section we prove Theorem [3.1. First, we examine the behavior of $u^{\varepsilon}$ for times which are small in the logarithmic scale.

Lemma 3.9. There is a positive $v_{0}$ such that for every $0<\delta<v_{0}$ there is $\varepsilon_{0}>0$ such that

$$
\left|u^{\varepsilon}\left(\exp \left(\lambda / \varepsilon^{2}\right), x\right)-g\left(x_{0}\right)\right| \leq \delta
$$

whenever $x \in D^{\delta}, 0<\varepsilon \leq \varepsilon_{0}$ and $\delta \leq \lambda \leq v_{0}$.

Proof. This lemma immediately follows from Lemma 3.3 .

The next three lemmas, central to the proof of Theorem 3.1, rule out certain types of behavior for the function $u^{\varepsilon}$.

Lemma 3.10. Suppose that $a_{1} \leq \mu_{n}<\lambda_{n} \leq a_{2}$ for some constants $a_{1}, a_{2}>0, \varepsilon_{n} \downarrow 0$ as $n \rightarrow \infty$, and

$$
u^{\varepsilon_{n}}\left(\exp \left(\mu_{n} / \varepsilon_{n}^{2}\right), x_{0}\right)=\beta_{1}, \quad u^{\varepsilon_{n}}\left(\exp \left(\lambda_{n} / \varepsilon_{n}^{2}\right), x_{0}\right)=\beta_{2}
$$

with $\beta_{1} \neq \beta_{2}$. Then there is $\delta>0$ such that

$$
\exp \left(\lambda_{n} / \varepsilon_{n}^{2}\right)-\exp \left(\mu_{n} / \varepsilon_{n}^{2}\right) \geq \exp \left(\delta / \varepsilon_{n}^{2}\right)
$$

for all large enough $n$.

Proof. Consider the process $X_{s}^{\lambda_{n}, x_{0}, \varepsilon_{n}}$ given by (19) with $\tau^{\varepsilon_{n}}$ being the first time when this process reaches the boundary of $D$. Define

$$
\bar{\tau}^{\varepsilon_{n}}=\min \left(\tau^{\varepsilon_{n}}, \exp \left(\lambda_{n} / \varepsilon_{n}^{2}\right)-\exp \left(\mu_{n} / \varepsilon_{n}^{2}\right)\right) .
$$

Then

$$
u^{\varepsilon_{n}}\left(\exp \left(\lambda_{n} / \varepsilon_{n}^{2}\right), x_{0}\right)=\mathrm{E} u^{\varepsilon_{n}}\left(\exp \left(\lambda_{n} / \varepsilon_{n}^{2}\right)-\bar{\tau}^{\varepsilon_{n}}, X_{\bar{\tau}^{\varepsilon_{n}}}^{\lambda_{n}, x_{0}, \varepsilon_{n}}\right) .
$$

The left hand side in this formula is equal to $\beta_{2}$. If (20) does not hold, then by Part (C) of Lemma 3.3 and Lemma 3.8 the right hand side can be made arbitrarily close to $\beta_{1}$ along a subsequence.

Lemma 3.11. Suppose that $a_{1} \leq \mu_{n}<\lambda_{n} \leq a_{2}$ for some constants $a_{1}, a_{2}>0, \varepsilon_{n} \downarrow 0$ as $n \rightarrow \infty$, and

$$
u^{\varepsilon_{n}}\left(\exp \left(\mu_{n} / \varepsilon_{n}^{2}\right), x_{0}\right)=\beta_{1}, \quad u^{\varepsilon_{n}}\left(\exp \left(\lambda_{n} / \varepsilon_{n}^{2}\right), x_{0}\right)=\beta_{2},
$$

If $g_{\min }<\beta_{1}<\beta_{2}<g_{\max }$, then neither of the following is possible:

(A) There is $\delta>0$ such that $\lambda_{n}<M\left(\beta_{2}\right)-\delta$,

(B) There is $\delta>0$ such that $G(c)<\beta_{2}-\delta$ for $c \in\left[\beta_{2}-\delta, \beta_{2}+\delta\right]$.

If $g_{\min }<\beta_{2}<\beta_{1}<g_{\max }$, then neither of the following is possible:

$\left(A^{\prime}\right)$ There is $\delta>0$ such that $\lambda_{n}<M\left(\beta_{2}\right)-\delta$,

$\left(B^{\prime}\right)$ There is $\delta>0$ such that $G(c)>\beta_{2}+\delta$ for $c \in\left[\beta_{2}-\delta, \beta_{2}+\delta\right]$. 
Proof. (A) Let us assume that $\lambda_{n}<M\left(\beta_{2}\right)-\delta$. We can find $\lambda_{n}^{\prime} \in\left[\mu_{n}, \lambda_{n}\right]$ such that $u^{\varepsilon_{n}}\left(\exp \left(\lambda_{n}^{\prime} / \varepsilon_{n}^{2}\right), x_{0}\right)=\beta_{2}$ and $u\left(t, x_{0}\right) \leq \beta_{2}$ for $t \in\left[\exp \left(\mu_{n} / \varepsilon_{n}^{2}\right), \exp \left(\lambda_{n}^{\prime} / \varepsilon_{n}^{2}\right)\right]$.

Let $\bar{\alpha}_{i j}(x)=a_{i j}\left(x, \beta_{2}\right)$. Let $\tau^{\varepsilon}$ be the first time when the process defined in (10) reaches the boundary of $D$. By part (B) of Lemma 3.2 , we can choose $\varkappa>0$ such that whenever $\alpha$ is Lipschitz continuous and satisfies (9) and (12), we have

$$
\mathrm{P}\left(\tau^{\varepsilon_{n}} \geq \exp \left(\lambda_{n}^{\prime} / \varepsilon_{n}^{2}\right)\right) \geq \mathrm{P}\left(\tau^{\varepsilon_{n}} \geq \exp \left(\left(M\left(\beta_{2}\right)-\delta\right) / \varepsilon_{n}^{2}\right)\right) \geq 1-\rho\left(\varepsilon_{n}\right),
$$

for $x \in D^{\delta}$, where $\rho$ does not depend on $\alpha$ and satisfies $\lim _{\varepsilon \downarrow 0} \rho(\varepsilon)=0$. Choose $\beta^{\prime}>0$ such that

$$
\left|a_{i j}\left(x, \beta_{2}\right)-a_{i j}(x, \beta)\right|<\varkappa
$$

whenever $\beta \in\left[\beta_{2}-2 \beta^{\prime}, \beta_{2}+2 \beta^{\prime}\right], x \in D^{\varkappa}$. Choose a sequence $\mu_{n}^{\prime} \in\left[\mu_{n}, \lambda_{n}^{\prime}\right]$ such that $u^{\varepsilon_{n}}\left(\mu_{n}^{\prime} / \varepsilon_{n}^{2}, x_{0}\right)=\beta_{2}-\beta^{\prime}$ and $u^{\varepsilon_{n}}\left(t, x_{0}\right) \in\left[\beta_{2}-\beta^{\prime}, \beta_{2}\right]$ for $t \in\left[\exp \left(\mu_{n}^{\prime} / \varepsilon_{n}^{2}\right), \exp \left(\lambda_{n}^{\prime} / \varepsilon_{n}^{2}\right)\right]$. By Lemma 3.8, we have

$$
\left|a_{i j}\left(x, \beta_{2}\right)-a_{i j}\left(x, u^{\varepsilon_{n}}(t, x)\right)\right|<\varkappa
$$

for $x \in D^{\varkappa}, t \in\left[\exp \left(\mu_{n}^{\prime} / \varepsilon_{n}^{2}\right), \exp \left(\lambda_{n}^{\prime} / \varepsilon_{n}^{2}\right)\right]$, if $\varepsilon_{n}$ is sufficiently small. Consider the process $X_{s}^{\lambda_{n}^{\prime}, x_{0}, \varepsilon_{n}}$ given by (19), with $\tau^{\varepsilon_{n}}$ now being the first time when this process reaches the boundary of $D$, and

$$
\bar{\tau}^{\varepsilon_{n}}=\min \left(\tau^{\varepsilon_{n}}, \exp \left(\lambda_{n}^{\prime} / \varepsilon_{n}^{2}\right)-\exp \left(\mu_{n}^{\prime} / \varepsilon_{n}^{2}\right)\right)
$$

Then

$$
u^{\varepsilon_{n}}\left(\exp \left(\lambda_{n}^{\prime} / \varepsilon_{n}^{2}\right), x_{0}\right)=\mathrm{E} u^{\varepsilon_{n}}\left(\exp \left(\lambda_{n}^{\prime} / \varepsilon_{n}^{2}\right)-\bar{\tau}^{\varepsilon_{n}}, X_{\bar{\tau}^{\varepsilon_{n}}}^{\lambda_{n}^{\prime}, x_{0}, \varepsilon_{n}}\right) .
$$

The left hand side in this formula is equal to $\beta_{2}$, while the right hand side can be made arbitrarily close to $\beta_{2}-\beta^{\prime}$ by considering sufficiently small $\varepsilon_{n}$ due to (22), Corollary 3.4 (which applies due to Lemma 3.10) and Lemma 3.8. This leads to a contradiction.

(B) Assume that $G(c)<\beta_{2}-\delta$ for $c \in\left[\beta_{2}-\delta, \beta_{2}+\delta\right]$. Let $\mathcal{A}$ be the set (consisting of either one or two points) where the minimum of $V^{a\left(\cdot, \beta_{2}\right)}\left(x_{0}, x\right)$ is attained. From the definition of $G$ it follows that $g(\mathcal{A}) \subset\left(-\infty, \beta_{2}-\delta\right)$. By part (C) of Lemma 3.2, we can choose $\varkappa>0$ such that whenever $\alpha$ is Lipschitz continuous and satisfies (9) and (12), we have

$$
\mathrm{P}\left(g\left(X_{\tau^{\varepsilon}}^{\sigma, x, \varepsilon}\right) \leq \beta_{2}-\delta / 2\right) \geq 1-\rho(\varepsilon)
$$

for $x \in D^{\delta}$ and all sufficiently small $\varepsilon$.

As in case (A), we can find $\beta^{\prime}>0$ and $\mu_{n} \leq \mu_{n}^{\prime}<\lambda_{n}^{\prime} \leq \lambda_{n}$ such that

$$
u^{\varepsilon_{n}}\left(\exp \left(\mu_{n}^{\prime} / \varepsilon_{n}^{2}\right), x_{0}\right)=\beta_{2}-\beta^{\prime}, \quad u^{\varepsilon_{n}}\left(\exp \left(\lambda_{n}^{\prime} / \varepsilon_{n}^{2}\right), x_{0}\right)=\beta_{2},
$$

and (23) holds for $x \in D^{\varkappa}, t \in\left[\exp \left(\mu_{n}^{\prime} / \varepsilon_{n}^{2}\right), \exp \left(\lambda_{n}^{\prime} / \varepsilon_{n}^{2}\right)\right]$, if $\varepsilon_{n}$ is sufficiently small. We can again employ formula (25) in which the left hand side is equal to $\beta_{2}$. The right hand side can be written as

$$
\mathrm{E} u^{\varepsilon_{n}}\left(\exp \left(\lambda_{n}^{\prime} / \varepsilon_{n}^{2}\right)-\bar{\tau}^{\varepsilon_{n}}, X_{\bar{\tau}^{\varepsilon}}^{\lambda_{n}^{\prime}, x_{0}, \varepsilon_{n}}\right)=
$$




$$
\mathrm{E}\left(\chi_{\left\{\bar{\tau}^{\varepsilon_{n}}<\tau^{\varepsilon}\right\}} u^{\varepsilon_{n}}\left(\exp \left(\mu_{n}^{\prime} / \varepsilon_{n}^{2}\right), X_{\bar{\tau}^{\varepsilon}}^{\lambda_{n}^{\prime}, x_{0}, \varepsilon_{n}}\right)\right)+\mathrm{E}\left(\chi_{\left\{\bar{\tau}^{\varepsilon_{n}}=\tau^{\varepsilon_{n}}\right\}} g\left(X_{\tau^{\varepsilon n}}^{\lambda_{n}^{\prime}, x_{0}, \varepsilon_{n}}\right)\right) .
$$

The first term in the right hand side here can be made arbitrarily close to $\mathrm{P}\left(\bar{\tau}^{\varepsilon_{n}}<\right.$ $\left.\tau^{\varepsilon_{n}}\right)\left(\beta_{2}-\beta^{\prime}\right)$ by Corollary 3.4 and Lemma 3.8. The second term on the right hand side can be estimated from above for large $n$ by $\mathrm{P}\left(\bar{\tau}^{\varepsilon_{n}}=\tau^{\varepsilon_{n}}\right)\left(\beta_{2}-\delta / 4\right)$ due to (26). This leads to a contradiction.

The proof of $\left(\mathrm{A}^{\prime}\right)$ and $\left(\mathrm{B}^{\prime}\right)$ is completely similar to the proof of $(\mathrm{A})$ and $(\mathrm{B})$.

Lemma 3.12. Suppose that $\lambda>0$. If $c_{1}>c_{0}$, then

$$
\liminf _{\varepsilon \downarrow 0}\left(u^{\varepsilon}\left(\exp \left(\lambda / \varepsilon^{2}\right), x_{0}\right)-c(\lambda)\right) \geq 0 .
$$

If $c_{1}<c_{0}$, then

$$
\limsup _{\varepsilon \downarrow 0}\left(u^{\varepsilon}\left(\exp \left(\lambda / \varepsilon^{2}\right), x_{0}\right)-c(\lambda)\right) \leq 0 .
$$

Proof. We shall only consider the first statement since the second one is completely similar. Note that

$$
\liminf _{\varepsilon \downarrow 0} u^{\varepsilon}\left(\exp \left(\lambda / \varepsilon^{2}\right), x_{0}\right) \geq c_{0} .
$$

Indeed, otherwise by Lemma 3.9 there are $\beta_{2}<\beta_{1}<c_{0}$ and sequences $v_{0} \leq \mu_{n}<\lambda_{n} \leq \lambda$ and $\varepsilon_{n} \downarrow 0$ such that (21) holds. Note that the graph of $G$ goes above the diagonal in a neighborhood of $c_{0}$, while $\beta_{1}$ and $\beta_{2}$ can be taken arbitrarily close to $c_{0}$. Therefore, Part $\left(\mathrm{B}^{\prime}\right)$ of Lemma 3.11 leads to a contradiction.

Thus, if (27) does not hold, then is there are $\delta>0$ and a sequence $\varepsilon_{n} \downarrow 0$ such that

$$
c_{0}-\delta<u^{\varepsilon_{n}}\left(\exp \left(\lambda / \varepsilon_{n}^{2}\right), x_{0}\right)<c(\lambda)-\delta .
$$

We choose $\delta$ sufficiently small so that the graph of $G$ goes above the diagonal on the interval $\left[c_{0}-2 \delta, c(\lambda)-\delta / 2\right]$. Take $\delta^{\prime}>0$ which will be specified later.

For each $c \in\left[c_{0}-\delta, c(\lambda)-\delta\right]$, by part (A) of Lemma 3.2, we can choose $\varkappa(c)>0$ such that whenever $\alpha$ is Lipschitz continuous and satisfies (91) and (12) with $\bar{\alpha}_{i j}(x)=a_{i j}(x, c)$ we have

$$
\begin{gathered}
\mathrm{P}\left(\tau^{\varepsilon} \leq \exp \left(\left(M(c)+\delta^{\prime}\right) / \varepsilon^{2}\right)\right) \geq 1-\rho(\varepsilon) . \\
\mathrm{P}\left(g\left(X_{\tau^{\varepsilon}}^{x_{0}, \varepsilon}\right) \geq \inf _{\bar{c} \in\left[c-\delta^{\prime}, c+\delta^{\prime}\right]} G(\bar{c})-\delta^{\prime}\right) \geq 1-\rho(\varepsilon)
\end{gathered}
$$

For each $c \in\left[c_{0}-\delta, c(\lambda)-\delta\right]$, find $l(c)<\delta^{\prime}$ such that $\left|a_{i j}(x, c)-a_{i j}(x, \bar{c})\right|<\varkappa(c)$ whenever $\bar{c} \in[c-l(c), c+l(c)], x \in D^{\varkappa(c)}$.

Choose a finite subcovering of the interval $\left[c_{0}-\delta, c(\lambda)-\delta\right]$ by the intervals $\left(c_{m}-\right.$ $\left.l\left(c_{m}\right) / 2, c_{m}+l\left(c_{m}\right) / 2\right)$ and take $l=\min _{m}\left(l\left(c_{m}\right)\right)$. Let $c_{0}-\delta=\beta_{0}<\beta_{1}<\ldots<\beta_{k}=c(\lambda)-\delta$ be such that $\beta_{i}-\beta_{i-1} \leq l / 10,1 \leq i \leq k$.

We claim that if $i \geq 1,0<\lambda^{\prime}<\lambda$ and $u^{\varepsilon_{n}}\left(\exp \left(\lambda / \varepsilon_{n}^{2}\right), x_{0}\right) \in\left[\beta_{i-1}, \beta_{i}\right]$ along a subsequence, then $u^{\varepsilon_{n}}\left(\exp \left(\lambda^{\prime} / \varepsilon_{n}^{2}\right), x_{0}\right) \leq \beta_{i-1}$ for large enough $n$ along the same subsequence. If this were not the case, then we would have

$$
u^{\varepsilon_{n}}\left(\exp \left(\lambda / \varepsilon_{n}^{2}\right), x_{0}\right) \in\left[\beta_{i-1}, \beta_{i}\right] \text { and } u^{\varepsilon_{n}}\left(\exp \left(\lambda^{\prime} / \varepsilon_{n}^{2}\right), x_{0}\right) \geq \beta_{i-1}
$$


along a further subsequence. Note that the function $u^{\varepsilon_{n}}\left(t, x_{0}\right)$ must take values in the interval $\left[\beta_{i-1}-l / 10, \beta_{i}+l / 10\right]$ for $t \in\left[\exp \left(\lambda^{\prime} / \varepsilon_{n}^{2}\right), \exp \left(\lambda / \varepsilon_{n}^{2}\right)\right]$, otherwise Part $\left(\mathrm{B}^{\prime}\right)$ of Lemma 3.11 leads to a contradiction.

By the construction above and Lemma 3.8, there is $m$ such that $\left|c_{m}-\beta_{i}\right|<\delta^{\prime}$ and

$$
\left|a_{i j}\left(x, c_{m}\right)-a_{i j}\left(x, u^{\varepsilon_{n}}(t, x)\right)\right|<\varkappa\left(c_{m}\right)
$$

for $x \in D^{\varkappa\left(c_{m}\right)}, t \in\left[\exp \left(\lambda^{\prime} / \varepsilon_{n}^{2}\right), \exp \left(\lambda / \varepsilon_{n}^{2}\right)\right]$, if $\varepsilon_{n}$ is sufficiently small.

Consider the process $X_{s}^{\lambda, x_{0}, \varepsilon_{n}}$ given by (19), with $\tau^{\varepsilon_{n}}$ being the first time when this process reaches the boundary of $D$, and

$$
\bar{\tau}^{\varepsilon_{n}}=\min \left(\tau^{\varepsilon_{n}}, \exp \left(\lambda / \varepsilon_{n}^{2}\right)-\exp \left(\lambda_{n}^{\prime} / \varepsilon_{n}^{2}\right)\right) .
$$

Then

$$
u^{\varepsilon_{n}}\left(\exp \left(\lambda / \varepsilon_{n}^{2}\right), x_{0}\right)=\operatorname{E} u^{\varepsilon_{n}}\left(\exp \left(\lambda / \varepsilon_{n}^{2}\right)-\bar{\tau}^{\varepsilon_{n}}, X_{\bar{\tau}^{\varepsilon}}^{\lambda, x_{0}, \varepsilon_{n}}\right) .
$$

The left hand side does not exceed $\beta_{i}$. If $\left(\exp \left(\lambda / \varepsilon_{n}^{2}\right)-\exp \left(\lambda^{\prime} / \varepsilon_{n}^{2}\right)\right) \geq \exp \left(\left(M\left(c_{m}\right)+\delta^{\prime}\right) / \varepsilon_{n}^{2}\right)$ (which is true if $\delta^{\prime}$ is sufficiently small and $n$ is sufficiently large), then from (29) and (30) it follows that the right hand side can be made larger than $\inf _{\bar{c} \in\left[c_{m}-\delta^{\prime}, c_{m}+\delta^{\prime}\right]} G(\bar{c})-2 \delta^{\prime}$. This leads to a contradiction if $\delta^{\prime}$ is small enough since $G$ is a piece-wise continuous function which stays above the diagonal on $\left[c_{0}-2 \delta, c(\lambda)-\delta / 2\right]$.

We have thus established that $u^{\varepsilon_{n}}\left(\exp \left(\lambda^{\prime} / \varepsilon_{n}^{2}\right), x_{0}\right) \leq \beta_{i-1}$. We can then extract a further subsequence such that $u^{\varepsilon_{n}}\left(\exp \left(\lambda^{\prime} / \varepsilon_{n}^{2}\right), x_{0}\right)$ belongs to one of the intervals $\left[\beta_{j-1}, \beta_{j}\right]$ with $j<i$. We can then take $\lambda^{\prime \prime}<\lambda^{\prime}$ and repeat the argument above to show that $u^{\varepsilon_{n}}\left(\exp \left(\lambda^{\prime \prime} / \varepsilon_{n}^{2}\right), x_{0}\right) \leq \beta_{j-1}$. After at most $k$ such steps, we obtain $\widetilde{\lambda}<\lambda$ such that $u^{\varepsilon_{n}}\left(\exp \left(\widetilde{\lambda} / \varepsilon_{n}^{2}\right), x_{0}\right) \leq \beta_{0}$ along a subsequence, and $\tilde{\lambda}$ can be chosen to be arbitrarily close to $\lambda$. This, however, is a contradiction with (28).

Proof of Theorem 3.1. By Lemma 3.8, it is sufficient to prove that

$$
\lim _{\varepsilon \downarrow 0} u^{\varepsilon}\left(\exp \left(\lambda / \varepsilon^{2}\right), x_{0}\right)=c(\lambda) .
$$

Case 1: $0<\lambda<M\left(c_{0}\right)$. Assume that

$$
\limsup _{\varepsilon \downarrow 0} u^{\varepsilon}\left(\exp \left(\lambda / \varepsilon^{2}\right), x_{0}\right)>c_{0}
$$

Take $c_{0}<\beta_{1}<\beta_{2}<\lim \sup _{\varepsilon \downarrow 0} u^{\varepsilon}\left(\exp \left(\lambda / \varepsilon^{2}\right), x_{0}\right)$ such that $M\left(\beta_{2}\right)>\lambda$. By Lemma 3.9, there are sequences $\varepsilon_{n} \downarrow 0$ and $v_{0} \leq \mu_{n}<\lambda_{n} \leq \lambda$ such that (21) holds. Thus Part (A) of Lemma 3.11 leads to a contradiction with (32). The inequality

$$
\liminf _{\varepsilon \downarrow 0} u^{\varepsilon}\left(\exp \left(\lambda / \varepsilon^{2}\right), x_{0}\right)<c_{0}
$$

can be ruled out in the same way by referring to Part $\left(\mathrm{A}^{\prime}\right)$ of Lemma 3.11 . 
Case 2: $\lambda \geq M\left(c_{0}\right), c_{1}=c_{0}$. Assume that

$$
\limsup _{\varepsilon \downarrow 0} u^{\varepsilon}\left(\exp \left(\lambda / \varepsilon^{2}\right), x_{0}\right)>c_{0} .
$$

Then, since $G$ is piece-wise continuous and passes from the left of the diagonal to the right of the diagonal at $c_{1}$, we can find $\delta>0$ and $\beta_{1}, \beta_{2}$ such that

$$
c_{0}<\beta_{1}<\beta_{2}<\limsup _{\varepsilon \downarrow 0} u^{\varepsilon}\left(\exp \left(\lambda / \varepsilon^{2}\right), x_{0}\right)
$$

and $G(c)<\beta_{2}-\delta$ for $c \in\left[\beta_{2}-\delta, \beta_{2}+\delta\right]$. By Lemma 3.9, there are sequences $\varepsilon_{n} \downarrow 0$ and $v_{0} \leq \mu_{n}<\lambda_{n} \leq \lambda$ such that (21) holds. Thus Part (B) of Lemma 3.11 leads to a contradiction with (33). The inequality

$$
\liminf _{\varepsilon \downarrow 0} u^{\varepsilon}\left(\exp \left(\lambda / \varepsilon^{2}\right), x_{0}\right)<c_{0}
$$

can be ruled out in the same way by referring to Part $\left(\mathrm{B}^{\prime}\right)$ of Lemma 3.11.

Case 3: $\lambda \geq M\left(c_{0}\right), c_{1}>c_{0}$. First assume that

$$
\limsup _{\varepsilon \downarrow 0} u^{\varepsilon}\left(\exp \left(\lambda / \varepsilon^{2}\right), x_{0}\right)>c(\lambda) .
$$

We can repeat the arguments of Case 2 to show that (34) implies that $c(\lambda)<c_{1}$. Then, since $\lambda$ is a point of continuity of $c(\lambda)$, we can find $\beta_{1}, \beta_{2}$ such that

$$
c(\lambda)<\beta_{1}<\beta_{2}<\limsup _{\varepsilon \downarrow 0} u^{\varepsilon}\left(\exp \left(\lambda / \varepsilon^{2}\right), x_{0}\right)
$$

and $M\left(\beta_{2}\right)>\lambda$. By Lemma [3.9, there are sequences $\varepsilon_{n} \downarrow 0$ and $v_{0} \leq \mu_{n}<\lambda_{n} \leq \lambda$ such that (21) holds. Thus Part (A) of Lemma 3.11 leads to a contradiction with (34).

Finally, from Lemma 3.12 it follows that

$$
\liminf _{\varepsilon \downarrow 0} u^{\varepsilon}\left(\exp \left(\lambda / \varepsilon^{2}\right), x_{0}\right) \geq c(\lambda) .
$$

Case 4: $\lambda \geq M\left(c_{0}\right), c_{1}<c_{0}$. This is completely similar to Case 3 .

Remark. If instead of the constant $\lambda$ in the argument of the function $u^{\varepsilon}$ in Theorem 3.1 , we have a positive function $\lambda(\varepsilon)$ such that $\lim _{\varepsilon \downarrow 0} \lambda(\varepsilon)=\lambda>0$, then

$$
\lim _{\varepsilon \downarrow 0} u^{\varepsilon}\left(\exp \left(\lambda(\varepsilon) / \varepsilon^{2}\right), x\right)=c(\lambda) .
$$

The proof of this statement requires only simple modifications to the proof of Theorem 3.1 , 


\section{Exit From the Domain}

Let the differential operator $L^{\varepsilon}$ and the function $G$ satisfy the assumptions of Section 3.1. Let $x \in D$ and $\lambda>0$. Recall that $X_{s}^{\lambda, x, \varepsilon}, s \in\left[0, \exp \left(\lambda / \varepsilon^{2}\right)\right]$, is the process defined in (19), with $\tau^{\varepsilon}$ being the first time when this process reaches the boundary of $D$. We put $\tau^{\varepsilon}=\infty$ on the event that the process does not reach the boundary by the time $\exp \left(\lambda / \varepsilon^{2}\right)$. Let $\bar{\tau}^{\varepsilon}=\min \left(\tau^{\varepsilon}, \exp \left(\lambda / \varepsilon^{2}\right)\right)$. Thus, if $\tau^{\varepsilon}<\infty$, then $X_{\bar{\tau}^{\varepsilon}}^{\lambda, x}$ is the location where the process first exits the domain. Let $\rho^{\varepsilon}$ be the measure on $\bar{D}$ induced by $X_{\bar{\tau}^{\varepsilon}}^{\lambda, x, \varepsilon}$ :

$$
\rho^{\varepsilon}(A)=\mathrm{P}\left(X_{\bar{\tau}^{\varepsilon}}^{\lambda, x, \varepsilon} \in A\right), \quad A \in \mathcal{B}(\bar{D}) .
$$

Let $\mu^{\varepsilon}$ be the restriction of $\rho^{\varepsilon}$ to $\partial D$ :

$$
\mu^{\varepsilon}(A)=\mathrm{P}\left(X_{\bar{\tau}^{\varepsilon}}^{\lambda, x, \varepsilon} \in A\right), \quad A \in \mathcal{B}(\partial D) .
$$

Note that $\mu^{\varepsilon}$ is not a probability measure, since $\mathrm{P}\left(X_{\bar{\tau}^{\varepsilon}}^{\lambda, x, \varepsilon} \in \partial D\right)<1$. In this section we shall examine the asymptotics of $\rho^{\varepsilon}$ and $\mu^{\varepsilon}$ when $\varepsilon \downarrow 0$.

We shall distinguish several cases corresponding to different values of $\lambda$. First consider the case when $0<\lambda<M\left(c_{0}\right)$.

Lemma 4.1. If $x \in D$ and $0<\lambda<M\left(c_{0}\right)$, then $\lim _{\varepsilon \downarrow 0} \mathrm{P}\left(\tau^{\varepsilon} \leq \exp \left(\lambda / \varepsilon^{2}\right)\right)=0$.

Proof. From Lemma 3.3 it follows that for $\delta>0$ there is $0<v_{0}<\lambda$ such that

$$
\lim _{\varepsilon \downarrow 0} \mathrm{P}\left(X_{s}^{v_{0}, x, \varepsilon} \in D \text { for all } 0 \leq s \leq \exp \left(v_{0} / \varepsilon^{2}\right)\right)=1
$$

uniformly in $x \in D^{\delta}$. We claim that for each $\varkappa>0$,

$$
\lim _{\varepsilon \downarrow 0} u^{\varepsilon}(t, x)=c_{0} \quad \text { uniformly in }(t, x) \in\left[\exp \left(v_{0} / \varepsilon^{2}\right), \exp \left(\lambda / \varepsilon^{2}\right)\right] \times D^{\varkappa} .
$$

Indeed, otherwise by Lemma 3.8 we could find sequences $\varepsilon_{n} \downarrow 0$ and $\lambda_{n} \in\left(v_{0}, \lambda\right)$ such that either $\limsup _{n \rightarrow \infty} u^{\varepsilon_{n}}\left(\exp \left(\lambda_{n} / \varepsilon_{n}^{2}\right), x_{0}\right)>c_{0}$ or $\liminf _{n \rightarrow \infty} u^{\varepsilon_{n}}\left(\exp \left(\lambda_{n} / \varepsilon_{n}^{2}\right), x_{0}\right)<c_{0}$. Suppose that the former is the case and that $c_{1}=c_{0}$ (the argument in the cases when $c_{1}>c_{0}$ and $c_{1}<c_{0}$ is similar). Then, from the conditions imposed on the function $G$ in Section 3.1 it follows that there are $c_{0}<\beta_{1}<\beta_{2}<\limsup _{n \rightarrow \infty} u^{\varepsilon_{n}}\left(\exp \left(\lambda_{n} / \varepsilon_{n}^{2}\right), x_{0}\right)$ such that the graph of $G$ goes below the diagonal in a neighborhood of the interval $\left[\beta_{1}, \beta_{2}\right]$. Moreover, there are $v_{0}<\mu_{n}^{\prime}<\lambda_{n}^{\prime}<\lambda_{n}$ such that $u^{\varepsilon_{n}}\left(\exp \left(\mu_{n}^{\prime} / \varepsilon_{n}^{2}\right), x_{0}\right)=\beta_{1}$ and $u^{\varepsilon_{n}}\left(\exp \left(\lambda_{n}^{\prime} / \varepsilon_{n}^{2}\right), x_{0}\right)=\beta_{2}$. This contradicts Part (B) of Lemma 3.11, thus establishing (38).

From (38) and Part (A) of Lemma 3.2 it follows that $\mathrm{P}\left(\tau^{\varepsilon} \leq \exp \left(\lambda / \varepsilon^{2}\right)-\exp \left(v_{0} / \varepsilon^{2}\right)\right)$ tends to zero as $\varepsilon \downarrow 0$. From Corollary 3.4 it then follows that $\mathrm{P}\left(X_{\exp \left(\lambda / \varepsilon^{2}\right)-\exp \left(v_{0} / \varepsilon^{2}\right)} \in D^{\delta}\right)$ tends to one as $\varepsilon \downarrow 0$. By the Markov property of the process and due to (37), this implies the statement of the lemma.

Next, let us examine the case when $\lambda>\lambda_{\max }$. (Recall that $\lambda_{\max }=\sup _{c \in\left[c_{0}, c_{1}\right]} M(c)$ if $c_{1} \geq c_{0}$ and $\lambda_{\max }=\sup _{c \in\left[c_{1}, c_{0}\right]} M(c)$ if $c_{1} \leq c_{0}$.) 
Lemma 4.2. Suppose that $x \in D$ and $\lambda>\lambda_{\max }$. If the minimum of the quasi-potential $\min _{x \in \partial D} V^{a\left(\cdot, c_{1}\right)}\left(x_{0}, x\right)$ is achieved at a single point $x^{*}\left(c_{1}\right)$, then $\mu^{\varepsilon}$ weakly converges to a probability measure $\mu$ concentrated at $x^{*}\left(c_{1}\right)$. If the minimum is achieved at two points $x_{1}^{*}\left(c_{1}\right)$ and $x_{2}^{*}\left(c_{1}\right)$ and $G_{1}\left(c_{1}\right) \neq G_{2}\left(c_{1}\right)$, then $\mu^{\varepsilon}$ weakly converges to a probability measure $\mu$ concentrated at those two points. In this case $\mu\left(x_{1}^{*}\left(c_{1}\right)\right) G_{1}\left(c_{1}\right)+\mu\left(x_{2}^{*}\left(c_{1}\right)\right) G_{2}\left(c_{1}\right)=c_{1}$.

Proof. Let $\lambda_{\max }<\lambda^{\prime}<\lambda$. Similarly to the proof of Lemma 4.1, and using the fact that $u^{\varepsilon}\left(\exp \left(\lambda / \varepsilon^{2}\right), x_{0}\right)$ converges to $c_{1}$ for each $\lambda>\lambda_{\max }$, we can show that

$$
\lim _{\varepsilon \downarrow 0} u^{\varepsilon}(t, x)=c_{1} \text { uniformly in }(t, x) \in\left[\exp \left(\lambda^{\prime} / \varepsilon^{2}\right), \exp \left(\lambda / \varepsilon^{2}\right)\right] \times D^{\varkappa},
$$

for each $\varkappa>0$. Let $\mathcal{A}=\left\{x^{*}\left(c_{1}\right)\right\}$ if the minimum $\min _{x \in \partial D} V^{a\left(\cdot, c_{1}\right)}\left(x_{0}, x\right)$ is achieved at a single point, and $\mathcal{A}=\left\{x_{1}^{*}\left(c_{1}\right), x_{2}^{*}\left(c_{1}\right)\right\}$ if the minimum is achieved at two points. Recall that $\tau^{\varepsilon}$ is the first time when the process $X_{s}^{\lambda, x, \varepsilon}$ reaches the boundary of $D$. From (39) and Lemma 3.2 it follows that

$$
\lim _{\varepsilon \downarrow 0} \mathrm{P}\left(\tau^{\varepsilon}<\exp \left(\lambda / \varepsilon^{2}\right)-\exp \left(\lambda^{\prime} / \varepsilon^{2}\right)\right)=1
$$

and for every $\delta>0$ we have

$$
\lim _{\varepsilon \downarrow 0} \mathrm{P}\left(\operatorname{dist}\left(X_{\tau^{\varepsilon} \wedge \exp \left(\lambda / \varepsilon^{2}\right)-\exp \left(\lambda^{\prime} / \varepsilon^{2}\right)}^{\lambda, x}, \mathcal{A}\right) \leq \delta\right)=1 .
$$

This immediately implies the desired result for the case of a single minimum point.

Let $U^{\delta}(y) \subseteq \partial D$ denote the $\delta$ neighborhood of a point $y$ on the boundary. In the case when the minimum is achieved at two points, we note that

$u^{\varepsilon}\left(\exp \left(\lambda / \varepsilon^{2}\right), x\right)=\operatorname{E} u^{\varepsilon}\left(\exp \left(\lambda / \varepsilon^{2}\right)-\tau^{\varepsilon} \wedge\left(\exp \left(\lambda / \varepsilon^{2}\right)-\exp \left(\lambda^{\prime} / \varepsilon^{2}\right)\right), X_{\tau^{\wedge} \wedge\left(\exp \left(\lambda / \varepsilon^{2}\right)-\exp \left(\lambda^{\prime} / \varepsilon^{2}\right)\right)}^{\lambda, \varepsilon}\right)$,

where the left hand side tends to $c_{1}$, while the right hand side is equal to

$$
\mu^{\varepsilon}\left(U^{\delta}\left(x_{1}^{*}\left(c_{1}\right)\right)\right) g\left(x_{1}^{*}\left(c_{1}\right)\right)+\mu^{\varepsilon}\left(U^{\delta}\left(x_{2}^{*}\left(c_{1}\right)\right)\right) g\left(x_{2}^{*}\left(c_{1}\right)\right)+\alpha(\varepsilon),
$$

where $\lim _{\varepsilon \downarrow 0} \alpha(\varepsilon)=0$, as follows from (40) and (41). It also follows from (40) and (41) that $\lim _{\varepsilon \downarrow 0} \mu^{\varepsilon}\left(\partial D \backslash\left(U^{\delta}\left(x_{1}^{*}\left(c_{1}\right)\right) \cup U^{\delta}\left(x_{2}^{*}\left(c_{1}\right)\right)\right)\right)=0$, which, together with (42), implies the desired result.

Finally, we consider the case when $c_{1} \neq c_{0}$ and the function $c(\lambda)$ is continuous at a point $\lambda \in\left(M\left(c_{0}\right), \lambda_{\max }\right)$. The cases $c_{1}<c_{0}$ and $c_{1}>c_{0}$ are completely similar to each other, so we shall only deal with the latter one. Let us introduce the needed notations. Fix $v_{0} \in\left(0, c_{0}\right)$. By Theorem 3.1, $\lim _{\varepsilon \downarrow 0} u^{\varepsilon}\left(\exp \left(v_{0} / \varepsilon^{2}\right), x_{0}\right)=c_{0}$ and $\lim _{\varepsilon \downarrow 0} u^{\varepsilon}\left(\exp \left(\lambda / \varepsilon^{2}\right), x_{0}\right)=$ $c(\lambda)>c_{0}$. For each $c \in\left[c_{0}, c(\lambda)\right]$ we define

$$
\lambda^{\varepsilon}(c)=\min \left(\inf \left\{\lambda^{\prime} \geq v_{0}: u^{\varepsilon}\left(\exp \left(\lambda^{\prime} / \varepsilon^{2}\right), x_{0}\right) \geq c\right\}, \lambda\right) .
$$


Let $\alpha^{\varepsilon}(c), c \in\left[c_{0}, c(\lambda)\right]$, be the probability that the process $X_{s}^{\lambda, x, \varepsilon}$ reaches the boundary of $D$ by the time $\exp \left(\lambda / \varepsilon^{2}\right)-\exp \left(\lambda^{\varepsilon}(c) / \varepsilon^{2}\right)$, that is

$$
\alpha^{\varepsilon}(c)=\mathrm{P}\left(\tau^{\varepsilon} \leq \exp \left(\lambda / \varepsilon^{2}\right)-\exp \left(\lambda^{\varepsilon}(c) / \varepsilon^{2}\right)\right)
$$

Since $\alpha^{\varepsilon}$ is left-continuous, it defines a measure $\nu^{\varepsilon}$ on $\mathcal{B}\left(\left[c_{0}, c(\lambda)\right]\right)$ via $\nu^{\varepsilon}([c, c(\lambda)])=$ $\alpha^{\varepsilon}(c)$. It will be important to identify the limit of $\alpha^{\varepsilon}$ as $\varepsilon \downarrow 0$. We define the function $\alpha:\left[c_{0}, c(\lambda)\right] \rightarrow[0,1]$ by:

$$
\alpha(c)=1-\exp \left(\int_{c}^{c(\lambda)} \frac{d z}{z-G(z)}\right) .
$$

Since $G$ is piece-wise continuous and its graph is above the diagonal in a neighborhood of $\left[c_{0}, c(\lambda)\right]$, the function $\alpha$ is a unique continuous function which satisfies the differential equation

$$
\alpha^{\prime}(c)=\frac{\alpha(c)-1}{G(c)-c}
$$

in the points of continuity of $G$ and the terminal condition $\alpha(c(\lambda))=0$. Notice that $\alpha(c) \in[0,1)$ for $c \in\left[c_{0}, c(\lambda)\right]$. The function $\alpha$ defines a measure $\nu$ on $\mathcal{B}\left(\left[c_{0}, c(\lambda)\right]\right)$ via $\nu([c, c(\lambda)])=\alpha(c)$.

Lemma 4.3. If $c_{1}>c_{0}, c(\lambda)$ is continuous at a point $\lambda \in\left(M\left(c_{0}\right), \lambda_{\max }\right)$, then

$$
\lim _{\varepsilon \downarrow 0} \alpha^{\varepsilon}(c)=\alpha(c)
$$

for $c \in\left[c_{0}, c(\lambda)\right]$.

Proof. Assume first that the minimum of the quasi-potential is achieved at a unique point $x^{*}(c)$ for each $c$ in a neighborhood of $\left[c_{0}, c(\lambda)\right]$, and therefore $G$ is a continuous function there. Take $\delta, \delta^{\prime}>0$ which will be specified later and $\delta^{\prime \prime}>0$ such that $x, x_{0} \in D^{\delta^{\prime \prime}}$. Let $\beta_{0}, \beta_{1}, \ldots, \beta_{k}$ be such that $\beta_{0}=c_{0}, \beta_{k}=c(\lambda)$ and $0<\beta_{i}-\beta_{i-1}<\delta, 1 \leq i \leq k$.

Consider the processes $Y_{s}^{i, x, \varepsilon}=X_{s}^{\lambda^{\varepsilon}\left(\beta_{i}\right), x, \varepsilon}, 1 \leq i \leq k-1$, and $Y_{s}^{k, x, \varepsilon}=X_{s}^{\lambda, x, \varepsilon}$. Let $\tau^{i, \varepsilon}$ be the first time when the process $Y_{s}^{i, x, \varepsilon}$ reaches the boundary of $D$. Let $B^{i, x, \varepsilon}, 1 \leq i \leq k-1$, be the event that $\tau^{i, \varepsilon} \leq \exp \left(\lambda^{\varepsilon}\left(\beta_{i}\right) / \varepsilon^{2}\right)-\exp \left(\lambda^{\varepsilon}\left(\beta_{i-1}\right) / \varepsilon^{2}\right)$, and $B^{k, x, \varepsilon}$ be the event that $\tau^{k, \varepsilon} \leq \exp \left(\lambda / \varepsilon^{2}\right)-\exp \left(\lambda^{\varepsilon}\left(\beta_{k-1}\right) / \varepsilon^{2}\right)$.

Using Lemma [3.2, it is not difficult to show that for each $\delta^{\prime}>0$ there is $\delta>0$ such that

$$
\lim _{\varepsilon \downarrow 0} \mathrm{P}\left(B^{i, x, \varepsilon} \cap\left\{\operatorname{dist}\left(Y_{\tau^{i, \varepsilon}}^{i, x, \varepsilon}, x^{*}\left(\beta_{i}\right)\right) \geq \delta^{\prime}\right\}\right)=0
$$

uniformly in $x \in D^{\delta^{\prime \prime}}$. Since $G$ is continuous, we can also make sure that $\delta$ is small enough so that

$$
\lim _{\varepsilon \downarrow 0} \mathrm{P}\left(B^{i, x, \varepsilon} \cap\left\{\left|g\left(Y_{\tau^{i, \varepsilon}}^{i, x, \varepsilon}\right)-G\left(\beta_{i}\right)\right| \geq \delta^{\prime}\right\}\right)=0
$$


uniformly in $x \in D^{\delta^{\prime \prime}}$. We can write $u^{\varepsilon}\left(\exp \left(\lambda / \varepsilon^{2}\right), x\right)$ in two different ways

$$
\begin{gathered}
u^{\varepsilon}\left(\exp \left(\lambda / \varepsilon^{2}\right), x\right)= \\
\operatorname{E} u^{\varepsilon}\left(\exp \left(\lambda / \varepsilon^{2}\right)-\tau^{\varepsilon} \wedge\left(\exp \left(\lambda / \varepsilon^{2}\right)-\exp \left(\lambda^{\varepsilon}\left(\beta_{i}\right) / \varepsilon^{2}\right)\right), X_{\tau^{\varepsilon} \wedge\left(\exp \left(\lambda / \varepsilon^{2}\right)-\exp \left(\lambda^{\varepsilon}\left(\beta_{i}\right) / \varepsilon^{2}\right)\right)}^{\lambda, x, \varepsilon}\right)
\end{gathered}
$$

and

$$
\begin{gathered}
u^{\varepsilon}\left(\exp \left(\lambda / \varepsilon^{2}\right), x\right)= \\
\operatorname{E} u^{\varepsilon}\left(\exp \left(\lambda / \varepsilon^{2}\right)-\tau^{\varepsilon} \wedge\left(\exp \left(\lambda / \varepsilon^{2}\right)-\exp \left(\lambda^{\varepsilon}\left(\beta_{i-1}\right) / \varepsilon^{2}\right)\right), X_{\tau^{\varepsilon} \wedge\left(\exp \left(\lambda / \varepsilon^{2}\right)-\exp \left(\lambda^{\varepsilon}\left(\beta_{i-1}\right) / \varepsilon^{2}\right)\right)}^{\lambda, x}\right) .
\end{gathered}
$$

Upon subtracting the right hand sides of these two equalities, using the Markov property, Corollary 3.4, Lemma 3.8 and (45), we obtain

$$
\beta_{i}\left(1-\alpha^{\varepsilon}\left(\beta_{i}\right)\right)=\left(\alpha^{\varepsilon}\left(\beta_{i-1}\right)-\alpha^{\varepsilon}\left(\beta_{i}\right)\right)\left(G\left(\beta_{i}\right)+h_{1}(i, \varepsilon)\right)+\beta_{i-1}\left(1-\alpha^{\varepsilon}\left(\beta_{i-1}\right)\right)+h_{2}(i, \varepsilon),
$$

where $h_{1}(i, \varepsilon) \leq \delta^{\prime}$ and $\lim _{\varepsilon \downarrow 0} h_{2}(i, \varepsilon)=0$. This implies the desired result once we recall that $\delta^{\prime}$ and $\delta$ can be taken arbitrarily small, since (46) shows that $\alpha^{\varepsilon}$ is a type of Euler's method approximation to the solution of (43).

The condition of continuity of $G$ can be easily removed once we recall that $G$ may have at most finitely many points of discontinuity.

Remark. Using similar arguments it is not difficult to show that

$$
\lim _{\varepsilon \downarrow 0} \mathrm{P}\left(\exp \left(\lambda / \varepsilon^{2}\right)-\exp \left(\lambda^{\varepsilon}\left(c_{0}\right) / \varepsilon^{2}\right)<\tau^{\varepsilon}<\infty\right)=0 .
$$

Moreover, using (46) and (44) it is possible to show that in order to find the limit of $\mu^{\varepsilon}$, one can take $\nu$, which is the limit of $\nu^{\varepsilon}$, and then take its push-forward by the function $x^{*}$ (since $\nu$ is an absolutely continuous measure, it is not essential that $x^{*}$ may be undefined in a finite number of points). The push-forward of $\nu$ will be denoted by $\mu$. Thus $\mu(A)=\nu\left(c \in\left[c_{0}, c(\lambda)\right]: x^{*}(c) \in A\right), A \in \mathcal{B}(\partial D)$.

Combining this with Lemmas 4.1 and 4.2 and Corollary 3.4, we can can formulate the following theorem.

Theorem 4.4. Let $\rho^{\varepsilon}$ and $\mu^{\varepsilon}$ be defined by (35) and (36), respectively. If $x \in D$ and $0<\lambda<M\left(c_{0}\right)$, then $\rho^{\varepsilon} \rightarrow \delta_{x_{0}}$, where $\delta_{x_{0}}$ is the probability measure concentrated at $x_{0}$ and $\mu^{\varepsilon} \rightarrow \mu$, where $\mu$ is the trivial measure, that is $\mu(\partial D)=0$.

If $\lambda>\lambda_{\max }$ and the minimum of the quasi-potential $\min _{x \in \partial D} V^{a\left(\cdot, c_{1}\right)}\left(x_{0}, x\right)$ is achieved at a single point $x^{*}\left(c_{1}\right)$, then $\rho^{\varepsilon}$ and $\mu^{\varepsilon}$ weakly converge to a probability measure $\mu$ concentrated at $x^{*}\left(c_{1}\right)$. If the minimum is achieved at two points $x_{1}^{*}\left(c_{1}\right)$ and $x_{2}^{*}\left(c_{1}\right)$ and $G_{1}\left(c_{1}\right) \neq G_{2}\left(c_{1}\right)$, then $\rho^{\varepsilon}$ and $\mu^{\varepsilon}$ weakly converge to a probability measure $\mu$ concentrated at those two points. Moreover, in this case $\mu\left(x_{1}^{*}\left(c_{1}\right)\right) G_{1}\left(c_{1}\right)+\mu\left(x_{2}^{*}\left(c_{1}\right)\right) G_{2}\left(c_{1}\right)=c_{1}$.

If $c_{1}>c_{0}$ and the function $c(\lambda)$ is continuous at a point $\lambda \in\left(M\left(c_{0}\right), \lambda_{\max }\right)$, then take the measure $\nu$ on $\mathcal{B}\left(\left[c_{0}, c(\lambda)\right]\right)$ defined via $\nu([c, c(\lambda)])=\alpha(c)$, where $\alpha$ is the solution of (43). The measures $\mu^{\varepsilon}$ weakly converge to the measure $\mu$ which is the push-forward of $\nu$ by the function $x^{*}$. The measures $\rho^{\varepsilon}$ weakly converge to the measure $c \delta_{x_{0}}+\mu$, where $c=1-\mu(\partial D)$. 
Remark. This theorem still holds if instead of $\lambda$ in the definition of $\mu^{\varepsilon}$ we have a positive function $\lambda(\varepsilon)$ such that $\lim _{\varepsilon \downarrow 0} \lambda(\varepsilon)=\lambda>0$.

Corollary 4.5. If $c_{1}>c_{0}$ and the function $c(\lambda)$ is continuous at $\lambda \in\left(M\left(c_{0}\right), \lambda_{\max }\right)$, then for every $\delta>0$ and $x \in D^{\delta}$ we have

$$
\lim _{\varepsilon \downarrow 0} u^{\varepsilon}\left(\exp \left(\lambda / \varepsilon^{2}\right), x\right)=\int_{c_{0}}^{c(\lambda)} g\left(x^{*}(c)\right) d \nu(c)+g\left(x_{0}\right)\left(1-\nu\left(\left[c_{0}, c(\lambda)\right]\right),\right.
$$

where $\nu$ is the measure on $\mathcal{B}\left(\left[c_{0}, c(\lambda)\right]\right)$ defined via $\nu([c, c(\lambda)])=\alpha(c)$.

Proof. The corollary immediately follows from Theorems 3.1 and 4.4 and the probabilistic representation of the solution to the initial-boundary value problem.

\section{$5 \quad$ Generalizations and Examples}

\subsection{The Case of a Nonlinear First Order Term}

We could allow the coefficient at the first order term to depend on $u^{\varepsilon}$ in (5)-(6):

$$
\begin{gathered}
\frac{\partial u^{\varepsilon}(t, x)}{\partial t}=L^{\varepsilon} u^{\varepsilon}:= \\
\frac{\varepsilon^{2}}{2} \sum_{i, j=1}^{d} a_{i j}\left(x, u^{\varepsilon}\right) \frac{\partial^{2} u^{\varepsilon}(t, x)}{\partial x_{i} \partial x_{j}}+\left(b\left(x, u^{\varepsilon}\right)+\varepsilon b_{1}\left(x, u^{\varepsilon}\right)\right) \cdot \nabla_{x} u^{\varepsilon}(t, x), \quad x \in D, \quad t>0, \\
u^{\varepsilon}(0, x)=g(x), x \in D, \quad u^{\varepsilon}(t, x)=g(x), \quad t \geq 0, x \in \partial D .
\end{gathered}
$$

All the assumptions made in Sections 2.1 and 3.1 remain in force, other than the following: instead of assuming that $b$ is a vector valued function on $D$, we assume that $b, b_{1} \in$ $C^{2}(D \times \mathbb{R})$, and there is a positive constant $k^{\prime}$ such that $(b(x, u), n(x))<-k^{\prime}$ for $x \in \partial D$, $u \in \mathbb{R}$, where $n(x)$ is the exterior normal to the boundary of $D$. Moreover, we assume that for each $u$ the vector field $b(\cdot, u)$ has a unique equilibrium point $x_{0}$ which does not depend on $u$ and that all the trajectories of the dynamical system $x^{\prime}(t)=b(x(t), u)$ starting in $D$ are attracted to $x_{0}$. We now assume that there is a smooth function $v$ defined on $\bar{D}$, such that $v\left(x_{0}\right)=0, v(x)>0$ for $x \neq x_{0}$, and $(b(x, u), \nabla v(x)) \leq-c\left|x-x_{0}\right|^{2}$ for some positive constant $c$, all $u$ and all $x$.

The definition of the function $M(c)$ from Section 3.1 needs to be modified to allow for the dependence of the drift term on a parameter. Namely, now

$$
M(c)=\min _{x \in \partial D} V^{a(\cdot, c), b(\cdot, c)}\left(x_{0}, x\right),
$$

where $V^{a(\cdot, c), b(\cdot, c)}$ is the quasi-potential for the process whose generator is equal to

$$
\frac{\varepsilon^{2}}{2} \sum_{i, j=1}^{d} a_{i j}(x, c) \frac{\partial^{2} u^{\varepsilon}(t, x)}{\partial x_{i} \partial x_{j}}+b(x, c) \cdot \nabla_{x} u^{\varepsilon}(t, x) .
$$


With this definition of $M(c)$, Theorems 3.1 and 4.4 remain valid, and the proofs do not require serious modifications.

\subsection{Metastable Distributions in the Case of Two Equilibrium Points}

In this section we again consider the solutions $u^{\varepsilon}$ to (15)-(66). Let all the assumptions about the domain $D$ and the operator $L^{\varepsilon}$ made in Sections 2.1 and 5.1 remain in force, except the following: instead of assuming the existence of a singe equilibrium point, we assume that there are two asymptotically stable equilibrium points $x_{1}, x_{2} \in D$ such that for almost every $x \in D$, with respect to the Lebesgue measure, the trajectory of (11) starting at $x$ is attracted to either $x_{1}$ or $x_{2}$. Let $D_{1} \subset D$ be the set of points in $D$ which are attracted to $x_{1}$ and $D_{2} \subset D$ the set of points attracted to $x_{2}$.

As before, we need to study the quasi-potential in order to determine the asymptotic behavior of $u^{\varepsilon}$. While in the case of a single equilibrium, the function $u^{\varepsilon}$ was nearly constant in $D^{\delta}$ at times of order $\exp \left(\lambda / \varepsilon^{2}\right)$ (Lemma 3.8), now $u^{\varepsilon}\left(\exp \left(\lambda / \varepsilon^{2}\right), x\right)$ will be close to $u^{\varepsilon}\left(\exp \left(\lambda / \varepsilon^{2}\right), x_{1}\right)$ for $x \in D_{1}^{\delta}$ and close to $u^{\varepsilon}\left(\exp \left(\lambda / \varepsilon^{2}\right), x_{2}\right)$ for $x \in D_{2}^{\delta}$. This explains why instead of freezing the second variable in the coefficients $a_{i j}$ in the right hand side of (5), the way it was done is Section 2.1, now we put the variable equal to $c_{1}$ in $D_{1}$ and $c_{2}$ in $D_{2}$. More precisely, for $c_{1}, c_{2} \in\left[g_{\min }, g_{\max }\right]$, let

$$
f_{c_{1}, c_{2}}(x)=c_{1} \chi_{D_{1}}(x)+c_{2} \chi_{D_{2}}(x)+\left(c_{1}+c_{2}\right) \chi_{D \backslash\left(D_{1} \cup D_{2}\right)}(x) / 2, \quad x \in D,
$$

where $\chi_{U}$ is the indicator function of a set $U \subseteq \mathbb{R}^{d}$. For a measurable positive-definite matrix-valued function $\alpha$ on $\bar{D}$, we define

$$
V^{\alpha}(x, y)=\inf _{T, \varphi}\left\{S_{0, T}^{\alpha}(\varphi): \varphi \in C([0, T], \bar{D}), \varphi(0)=x, \varphi(T)=y\right\}, \quad x, y \in \bar{D},
$$

where the normalized action functional $S$ was defined in Section 2.2. Instead of function $M$ used in Section 2.1, we now have functions $M_{x_{1}, x_{2}}, M_{x_{2}, x_{1}}, M_{x_{1}, \partial D}$ and $M_{x_{2}, \partial D}$. These are defined by

$$
\begin{aligned}
M_{x_{1}, x_{2}}\left(c_{1}\right) & =V^{a\left(\cdot, f_{c_{1}, c_{2}}(\cdot)\right)}\left(x_{1}, x_{2}\right), \\
M_{x_{2}, x_{1}}\left(c_{2}\right) & =V^{a\left(\cdot, f_{c_{1}, c_{2}}(\cdot)\right)}\left(x_{2}, x_{1}\right), \\
M_{x_{1}, \partial D}\left(c_{1}, c_{2}\right) & =\min _{x \in \partial D} V^{a\left(\cdot, f_{c_{1}, c_{2}}(\cdot)\right)}\left(x_{1}, x\right), \\
M_{x_{2}, \partial D}\left(c_{1}, c_{2}\right) & =\min _{x \in \partial D} V^{a\left(\cdot, f_{c_{1}, c_{2}}(\cdot)\right)}\left(x_{2}, x\right) .
\end{aligned}
$$

It is not difficult to check that the right hand side of (49) does not depend on $c_{2}$ and the right hand side of (50) does not depend on $c_{1}$. For the process governed by equation (5), with $a_{i j}\left(\cdot, f_{c_{1}, c_{2}}(\cdot)\right)$ instead of the nonlinear coefficients $a_{i j}\left(x, u^{\varepsilon}\right)$, the transition from $x_{1}$ to a small neighborhood of $x_{2}$ occurs in time of order $\exp \left(M_{x_{1}, x_{2}}\left(c_{1}\right) / \varepsilon^{2}\right)$ (provided that the process does not exit the domain $D$ earlier). Similarly, the transition from 
$x_{2}$ to a neighborhood of $x_{1}$ occurs in time of order $\exp \left(M_{x_{2}, x_{1}}\left(c_{2}\right) / \varepsilon^{2}\right)$, while the transition from $x_{1}$ and $x_{2}$ to the boundary occurs in time of order $\exp \left(M_{x_{1}, \partial D}\left(c_{1}, c_{2}\right) / \varepsilon^{2}\right)$ and $\exp \left(M_{x_{2}, \partial D}\left(c_{1}, c_{2}\right) / \varepsilon^{2}\right)$, respectively.

In this section we would like to study the equation at a time scale which is sufficiently large for the process to make excursions between the neighborhoods of $x_{1}$ and $x_{2}$ and back, yet not too large so that the process starting at $x_{1}$ or $x_{2}$ does not exit the domain. Therefore we assume that

$$
\max \left(\sup _{c_{1} \in\left[g_{\min }, g_{\max }\right]} M_{x_{1}, x_{2}}\left(c_{1}\right), \sup _{c_{2} \in\left[g_{\min }, g_{\max }\right]} M_{x_{2}, x_{1}}\left(c_{2}\right)\right)<M^{\partial}
$$

where

$$
M^{\partial}=\min \left(\inf _{c_{1}, c_{2} \in\left[g_{\min }, g_{\max }\right]} M_{x_{1}, \partial D}\left(c_{1}, c_{2}\right), \inf _{c_{1}, c_{2} \in\left[g_{\min }, g_{\max }\right]} M_{x_{2}, \partial D}\left(c_{1}, c_{2}\right)\right) .
$$

For example, if $a$ and $b$ are defined in the entire space $\mathbb{R}^{d}, a$ is bounded and $b$ satisfies $(b(x), x) \leq A-B(x, x)$ for some positive constants $A$ and $B$, then this condition will be satisfied for any domain $D$ which contains a sufficiently large ball centered at the origin.

Let $c_{1}=g\left(x_{1}\right)$ and $c_{2}=g\left(x_{2}\right)$. Without loss of generality we may assume that $c_{1} \leq c_{2}$. In order to formulate the results on the asymptotics of $u^{\varepsilon}$, we need to introduce functions $c^{1}(\lambda)$ and $c^{2}(\lambda)$ which are similar to the function $c(\lambda)$ from in Section 3.1 .

Let $0<\lambda<M^{\partial}$, and define $c^{1}(\lambda)$ as follows:

For $0<\lambda<M_{x_{1}, x_{2}}\left(c_{1}\right)$, let $c^{1}(\lambda)=c_{1}$.

For $\lambda \geq M_{x_{1}, x_{2}}\left(c_{1}\right)$, let $c^{1}(\lambda)=\min \left\{c_{2}, \min \left\{c: c \in\left[c_{1}, c_{2}\right], M_{x_{1}, x_{2}}(c)=\lambda\right\}\right\}$. Similarly, we define $c^{2}(\lambda)$ as follows:

For $0<\lambda<M_{x_{2}, x_{1}}\left(c_{2}\right)$, let $c^{2}(\lambda)=c_{2}$.

For $\lambda \geq M_{x_{2}, x_{1}}\left(c_{2}\right)$, let $c^{2}(\lambda)=\max \left\{c_{1}, \max \left\{c: c \in\left[c_{1}, c_{2}\right], M_{x_{2}, x_{1}}(c)=\lambda\right\}\right\}$.

Let $\lambda^{*}=\inf \left\{\lambda: c^{1}(\lambda) \geq c^{2}(\lambda)\right\}$. Assume that at least one of the functions $c^{1}$ and $c^{2}$ is continuous at $\lambda^{*}$. Let $c^{*}=c^{1}\left(\lambda^{*}\right)$ if $c^{1}$ is continuous at $\lambda^{*}$ and $c^{*}=c^{2}\left(\lambda^{*}\right)$ otherwise. Let $\bar{c}^{1}(\lambda)=\min \left(c^{1}(\lambda), c^{*}\right)$ and $\bar{c}^{2}(\lambda)=\max \left(c^{2}(\lambda), c^{*}\right)$.

We can now formulate the following analog of Theorem 3.1

Theorem 5.1. Let the above assumptions be satisfied. Suppose that the function $\bar{c}^{1}(\lambda)$ is continuous at a point $\lambda \in\left(0, M^{\partial}\right)$. Then for every $\delta>0$ the following limit

$$
\lim _{\varepsilon \downarrow 0} u^{\varepsilon}\left(\exp \left(\lambda / \varepsilon^{2}\right), x\right)=\bar{c}^{1}(\lambda)
$$

is uniform in $x \in D_{1}^{\delta}$. Suppose that the function $\bar{c}^{2}(\lambda)$ is continuous at a point $\lambda \in\left(0, M^{\partial}\right)$. Then for every $\delta>0$ the following limit

$$
\lim _{\varepsilon \downarrow 0} u^{\varepsilon}\left(\exp \left(\lambda / \varepsilon^{2}\right), x\right)=\bar{c}^{2}(\lambda)
$$

is uniform in $x \in D_{2}^{\delta}$. 
Remark. If $\lambda>\lambda^{*}$, then $\bar{c}^{1}(\lambda)=\bar{c}^{2}(\lambda)=c^{*}$. It is not difficult to see that the limit

$$
\lim _{\varepsilon \downarrow 0} u^{\varepsilon}\left(\exp \left(\lambda / \varepsilon^{2}\right), x\right)=c^{*}
$$

is uniform in $(x, \lambda) \in D^{\delta} \times[\bar{\lambda}, \infty)$ for each $\bar{\lambda}>\lambda^{*}$. Therefore, for each $\delta>0$ and $\bar{\lambda}>\lambda^{*}$ there is $\varepsilon_{0}>0$ such that

$$
\left|u^{\varepsilon}(t, x)-c^{*}\right| \leq \delta
$$

whenever $\varepsilon \in\left(0, \varepsilon_{0}\right), x \in D^{\delta}$ and $t \geq \exp \left(\bar{\lambda} / \varepsilon^{2}\right)$.

Recall that $X_{s}^{\lambda, x, \varepsilon}, s \in\left[0, \exp \left(\lambda / \varepsilon^{2}\right)\right]$, is the process defined in (19), $\tau^{\varepsilon}$ is the first time when this process reaches the boundary of $D$ and $\bar{\tau}^{\varepsilon}=\min \left(\tau^{\varepsilon}, \exp \left(\lambda / \varepsilon^{2}\right)\right)$. Since we assume that $\lambda<M^{\partial}$, the probability that $\tau^{\varepsilon}<\exp \left(\lambda / \varepsilon^{2}\right)$ now tends to zero as $\varepsilon \downarrow 0$. The distribution of the random variable $X_{\bar{\tau}^{\varepsilon}}^{\lambda, x, \varepsilon}$ will be concentrated near the points $x_{1}$ and $x_{2}$.

Theorem 5.2. Suppose that $c_{1} \neq c_{2}$. If the function $\bar{c}^{1}(\lambda)$ is continuous at a point $\lambda \in\left(0, M^{\partial}\right)$ and $x \in D_{1}$, then the distribution of the random variable $X_{\bar{\tau}^{\varepsilon}}^{\lambda, x, \varepsilon}$ converges to the measure $\mu_{1}^{\lambda}=a_{1} \delta_{x_{1}}+a_{2} \delta_{x_{2}}$, where the coefficients $a_{1}$ and $a_{2}$ can be found from the equations $\bar{c}^{1}(\lambda)=a_{1} c_{1}+a_{2} c_{2}, a_{1}+a_{2}=1$.

If the function $\bar{c}^{2}(\lambda)$ is continuous at a point $\lambda \in\left(0, M^{\partial}\right)$ and $x \in D_{2}$, then the distribution of the random variable $X_{\bar{\tau}^{\varepsilon}}^{\lambda, x}$ converges to the measure $\mu_{2}^{\lambda}=a_{1} \delta_{x_{1}}+a_{2} \delta_{x_{2}}$, where the coefficients $a_{1}$ and $a_{2}$ can be found from the equations $\bar{c}^{2}(\lambda)=a_{1} c_{1}+a_{2} c_{2}$, $a_{1}+a_{2}=1$.

If $\lambda \in\left(\lambda^{*}, M^{\partial}\right)$ and $x \in D$, then the distribution of the random variable $X_{\bar{\tau}^{\varepsilon}}^{\lambda, x, \varepsilon}$ converges to the measure $\mu^{*}=a_{1} \delta_{x_{1}}+a_{2} \delta_{x_{2}}$, where the coefficients $a_{1}$ and $a_{2}$ can be found from the equations $c^{*}=a_{1} c_{1}+a_{2} c_{2}, a_{1}+a_{2}=1$.

The proofs of Theorems 5.1 and 5.2 rely on the same techniques as those used in the proofs of Theorems 3.1 and 4.4, and therefore will not be presented here. If $a$ is bounded and $b$ satisfies $(b(x), x) \leq A-B(x, x)$ for some positive constants $A$ and $B$, then similar results can be formulated for the Cauchy problem in $\mathbb{R}^{d}$ and the corresponding nonlinear perturbations of the dynamical system in $\mathbb{R}^{d}$. In this case we do not need the condition $\lambda<M^{\partial}$, but can instead consider all $\lambda \in(0, \infty)$.

\subsection{Examples}

In this section we give two examples when we can easily calculate the function $M(c)$ defined in Section 3.1.

In the first example, we assume that the domain $D$ is one dimensional: $D=(A, B)$. We assume that $a(x, u) \in C^{2}([A, B] \times \mathbb{R})$ is positive, $b(x, u) \in C^{2}([A, B] \times \mathbb{R}), \partial b(x, u) / \partial x<$ $k<0$ and there is a point $x_{0} \in(A, B)$ such that $b\left(x_{0}, u\right)=0$ for all $u$. In this case the operator

$$
L^{\varepsilon} u=\frac{\varepsilon^{2}}{2} a(x, u) u^{\prime \prime}+b(x, u) u^{\prime}
$$


satisfies the assumptions of Section 5.1. The quasi-potential, which will now be denoted by $V^{c}$, is given by

$$
V^{c}\left(x_{0}, x\right)=-2 \int_{x_{0}}^{x} \frac{b(y, c)}{a(y, c)} d y
$$

as is easily seen from the definition of the action functional (see Section 2.2). Therefore,

$$
M(c)=\min \left(V^{c}\left(x_{0}, A\right), V^{c}\left(x_{0}, B\right)\right)=\min \left(-2 \int_{x_{0}}^{A} \frac{b(y, c)}{a(y, c)} d y,-2 \int_{x_{0}}^{B} \frac{b(y, c)}{a(y, c)} d y\right) .
$$

The function $G(c)$ may take at most two values: $g(A)$ and $g(B)$. In particular, the value $g(A)$ is taken on the set $\left\{c: V^{c}\left(x_{0}, A\right)<V^{c}\left(x_{0}, B\right)\right\}$, while the value $g(B)$ is taken on the set $\left\{c: V^{c}\left(x_{0}, A\right)>V^{c}\left(x_{0}, B\right)\right\}$.

Picture 2 shows an example of the graphs of functions $M(c)$ and $G(c)$ in the case when $g(A)=\inf _{x \in[A, B]} g(x)<\sup _{x \in[A, B]} g(x)=g(B)$. From Theorem 4.4 and the discussion in Section 5.1 it follows that for $\lambda>\lambda_{\max }$, the distribution of the random variable $X_{\bar{\tau}^{\varepsilon}}^{\lambda, x, \varepsilon}$ converges to the probability measure $\mu$ concentrated at the end points of the segment. This measure can be found from the relations

$$
\mu(A) g(A)+\mu(B) g(B)=c_{1}, \quad \mu(A)+\mu(B)=1 .
$$

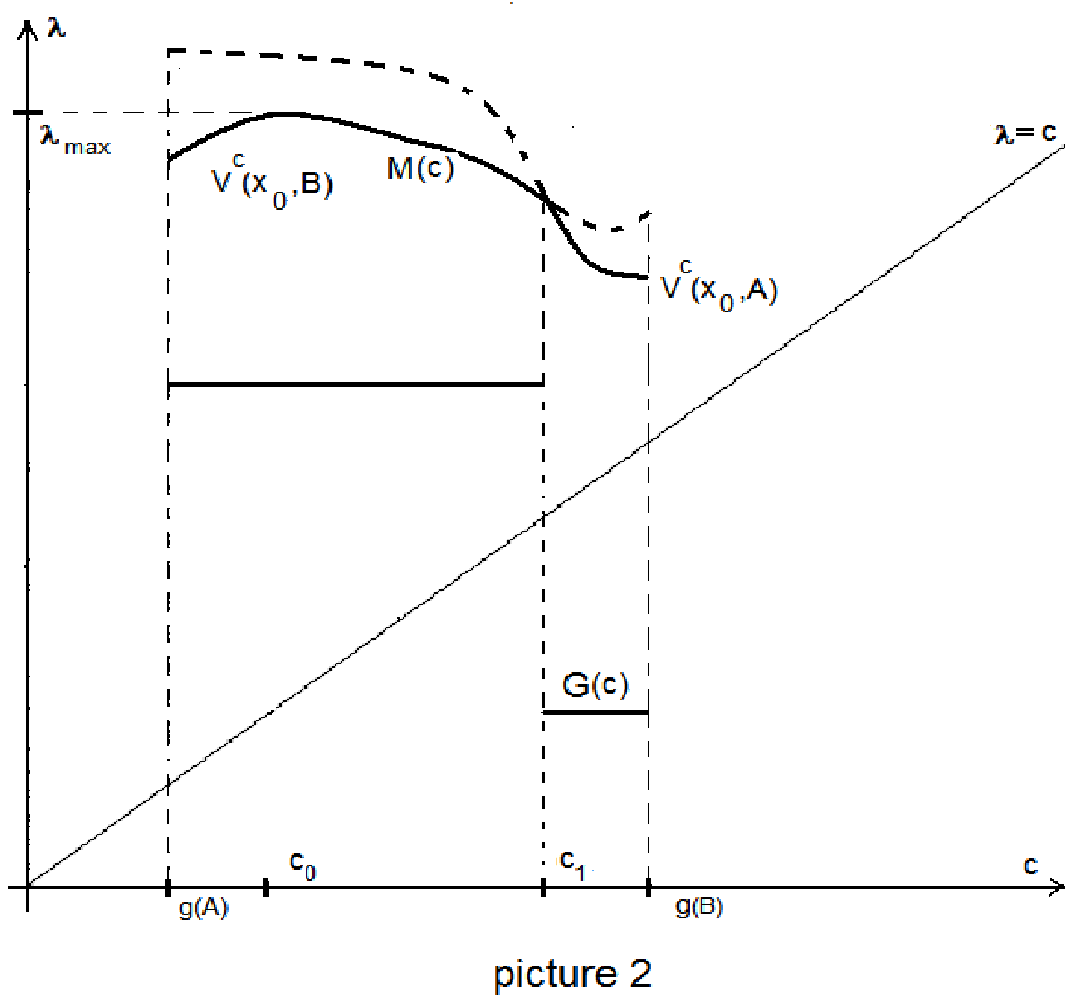


In the second example we assume that $D \subset \mathbb{R}^{d}$ contains the origin $x_{0}=0$. Let the operator $L^{\varepsilon}$ be as follows

$$
L^{\varepsilon} u=\frac{\varepsilon^{2}}{2} \sum_{i, j=1}^{d} a_{i j}(u) \frac{\partial^{2} u(t, x)}{\partial x_{i} \partial x_{j}}+(A(u) x) \cdot \nabla u,
$$

where $a$ is a positive-definite matrix which depends smoothly on $u$, and $A$ is a matrix with negative eigenvalues which depends smoothly on $u$. As has been demonstrated in [3], the quasi-potential, which we shall denote by $V^{c}$, is given by the quadratic form

$$
V^{c}\left(x_{0}, x\right)=\frac{1}{2}\left(B^{-1}(c) x, x\right)
$$

where the matrix $B$ is given by

$$
B(c)=\int_{0}^{\infty} \exp (A(c) t) a(c) \exp \left(A^{*}(c) t\right) d t
$$

Therefore, $M(c)=\min _{x \in \partial D}\left(B^{-1}(c) x, x\right) / 2$.

Acknowledgements: While working on this article, M. Freidlin was supported by NSF grant DMS-0803287 and L. Koralov was supported by NSF grant DMS-0706974.

\section{References}

[1] Aronson D.G., Non-negative solutions of linear parabolic equations, Ann. Sci. Norm. Sup. Pisa 22 (1968), 607-694.

[2] Athreya A., Freidlin M.I., Metastability and Stochastic Resonance in NearlyHamiltonian Systems, Stochastics and Dynamics, 8, 1, pp 1-21, 2008.

[3] Chen Z., Freidlin M.I., Smoluchowski-Kramers Approximation and Exit Problems, Stochastics and Dynamics, 5, pp 569-585, 2005.

[4] Freidlin M. I., Metastability and Stochastic Resonance of Multiscale Systems, Contemporary Mathematics, 2008.

[5] Freidlin M. I., Sublimiting Distributions and Stabilization of Solutions of Parabolic Equations with a Small Parameter, Soviet Math. Dokl., 235, 5, pp 1042-1045, 1977.

[6] Freidlin M. I., Quasi-deterministic Approximation, Metastability and Stochastic Resonance, Physica D, 137, pp 333-352, 2000. 
[7] Freidlin M.I., Koralov L., Metastability for Nonlinear Random Perturbations of Dynamical Systems, Stochastic Processes and Applications 120 (2010), no. 7, 1194-1214.

[8] Freidlin M. I., Wentzell A. D., Random Perturbations of Dynamical Systems, Springer 1998.

[9] Krylov N.V., Nonlinear Elliptic and Parabolic Equations of the Second Order (Mathematics and its Applications), Springer 1987.

[10] Oliveiri E., Vares M.E. Large Deviations and Metastability, Cambridge University Press, 2005. 